\title{
Asymmetric organocatalytic synthesis of substituted chiral 1,4-
}

\section{dihydropyridine derivatives}

Fernando Auria-Luna, ${ }^{1}$ Eugenia Marqués-López, ${ }^{1, *}$ M. Concepción Gimeno, ${ }^{2}$ Roghayeh

Heiran, ${ }^{1}$ Somayeh Mohammadi, ${ }^{1}$ and Raquel P. Herrera ${ }^{1, *}$

${ }^{1}$ Laboratorio de Organocatálisis Asimétrica, Departamento de Química Orgánica. Instituto de Síntesis Química y Catálisis Homogénea (ISQCH), CSIC-Universidad de Zaragoza. C/ Pedro Cerbuna 12, E-50009 Zaragoza, Spain.

${ }^{2}$ Departamento de Química Inorgánica. Instituto de Síntesis Química y Catálisis Homogénea (ISQCH), CSIC-Universidad de Zaragoza. C/ Pedro Cerbuna, Nº12, E-50009 Zaragoza, Spain.

\begin{abstract}

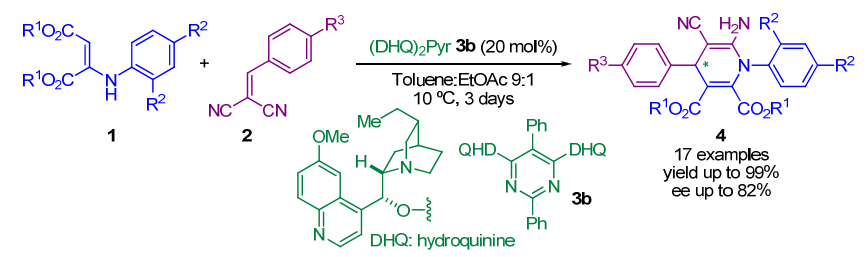

The first cinchona alkaloid organocatalyzed enantioselective synthesis of chiral 1,4dihydropyridine derivatives is described. Bis-cinchona catalyst $\mathbf{3 b}$ activates the Michael addition reaction between malononitrile derivatives $\mathbf{2}$ and enamines $\mathbf{1}$ affording the appealing and highly substituted 1,4-dihydropyridines $\mathbf{4}$ with very good results in most cases. This is one of very few examples of the synthesis of chiral 1,4-dihydropyridines by a catalytic procedure. The highly substituted final compounds are of interest for their potential biological activity. This efficient protocol opens the door to a new area of research for the asymmetric construction of these interesting skeletons for which enantioselective syntheses are still very limited.
\end{abstract}


Keywords: 1,4-Dihydropyridine; Asymmetric Organocatalysis; Cinchona alkaloid; Enamine; Malononitrile

\section{Introduction}

1,4-Dihydropyridine derivatives (1,4-DHPs) are a significant class of pharmacophore compounds frequently found in natural products and, more recently, even as biomimetic reducing agents. ${ }^{1}$ Their interesting biological properties have allowed them to find medicinal application in the treatments of different diseases. ${ }^{2}$ In particular, their biological importance has been demonstrated by their use as vasodilators, antihypertensive, anti-inflammatory, antihypoxic, anti-ischemic and antitubercular agents, and above all as calcium channel modulators (Figure 1). ${ }^{2}$ Interestingly, as with other drug molecules, the role of the stereochemistry at C-4 can exhibit both qualitative and quantitative differences in the biological activity of these compounds. Thus, the control of the stereoselectivity of these chiral centers becomes an inspiring task in the field of enantioselective organocatalysis. ${ }^{3}$<smiles>COC(=O)C1=C(C)NC(C)=C(C(OC)OC)C1c1ccccc1[N+](=O)[O-]</smiles>

Nifedipine<smiles>CCOC(=O)C1=C(C)NC(C)=C(C(C)=O)C1c1cccc([N+](=O)[O-])c1</smiles>

Nitrendipine<smiles>CCOC(=O)C1=C(C)NC(C)=C(C(C)=O)C1c1cccc(Cl)c1Cl</smiles>

Felodipine

Figure 1. Representative structures of 1,4-dihydropyridine based drugs.

1,4-DHPs are generally synthesized following the classical four-component Hantzsch reaction, ${ }^{4}$ which involves the condensation of an aldehyde, two equivalents of a $\beta$-ketoester and ammonia or a synthetic equivalent in a multicomponent approach. ${ }^{5}$ The synthesis of racemic $1,4-\mathrm{DHPs}$ is an active task in organic chemistry, ${ }^{6}$ and chiral analogues have mainly been obtained either by the use of chiral auxiliaries ${ }^{3}$ or by chiral resolution. ${ }^{7}$ However, very few enantioselective organocatalytic methods are known. ${ }^{8}$ This fact together with the 
biological interest of these molecules and the search for new analogues with novel binding properties, encouraged us to develop a new approach for their asymmetric synthesis via chiral organocatalysis. ${ }^{9}$ We envisioned that chiral organic bases such as cinchona derivatives could promote the enantioselective version of this reaction, since $\mathrm{Et}_{3} \mathrm{~N}$ is able to promote the racemic formation of 1,4-DHPs. ${ }^{10}$ Herein, we report our results concerning the synthesis of the highly functionalized 1,4-DHPs $\mathbf{4}$ via reaction of enamines $\mathbf{1}$ and malononitriles $\mathbf{2}$ in the presence of chiral basic organocatalysts 3 (Scheme 1).

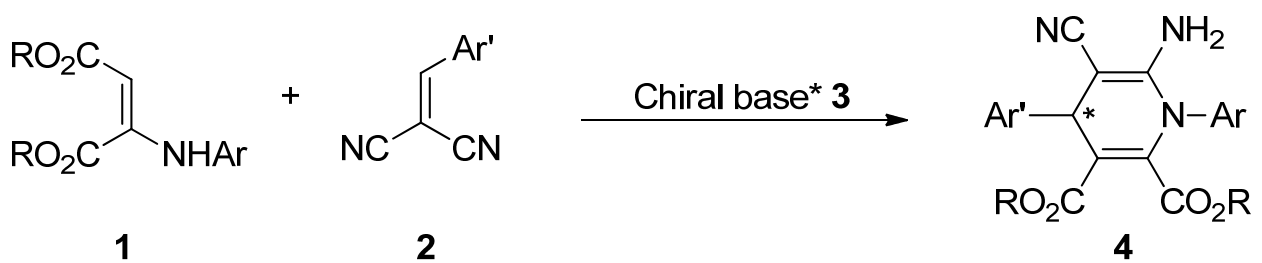

Scheme 1. Model reaction for the synthesis of chiral 1,4-DHPs 4.

\section{Results and Discussion}

To explore the feasibility of this approach, we studied the capacity of different chiral basic organocatalysts 3a-i to promote the reaction (Figure 2). 
<smiles>Oc1nnc(O)c2ccccc12</smiles>

$(\mathrm{DHQ})_{2}$ Phal

3a<smiles></smiles>

(DHQD) ${ }_{2}$ Phal

3d<smiles>Oc1nc(-c2ccccc2)nc(O)c1-c1ccccc1</smiles>

(DHQ) $)_{2} \mathrm{Pyr}$

$3 b$<smiles>O=Cc1nc(-c2ccccc2)nc(O[OH2+])c1-c1ccccc1</smiles>

(DHQD) ${ }_{2} \mathrm{Pyr}$

$3 e$<smiles>CCC1CN2CCC1[C@H]([C@H](OC)c1ccnc3ccc(OC)cc13)N2</smiles><smiles>O=C1c2ccccc2C(=O)c2c(O)ccc(O)c21</smiles>

$(\mathrm{DHQ})_{2} \mathrm{ANQ}$ 3c<smiles></smiles>

$(\mathrm{DHQD})_{2} \mathrm{ANQ}$

$3 f$<smiles>CCC1C2CCN1C[C@H]2[C@H](OC)c1ccnc2ccc(OC)cc12</smiles>

DHQD<smiles>[R]c1ccc2nccc([C@@H](O)[C@H]3CN4CC[C@H]3C4C=C)c2c1</smiles>

QN 3g R = OMe $\mathrm{CD} 3 \mathrm{~h} \mathrm{R}=\mathrm{H}$

Figure 2. Cinchona alkaloids 3a-i tested as catalysts.

We examined the reactivity and enantioselectivity provided by these catalysts in a model reaction between enamines 1a-b and malononitrile $\mathbf{2 a}$. A selection of these results is reported in Table 1.

Table 1. Screening of the reaction conditions for the synthesis of chiral 1,4-DHPs 4aa-ba. ${ }^{a}$<smiles>[R]OC(=O)C=C(Nc1ccc(C=C(C#N)C#N)cc1)Nc1ccc(OC)cc1OC</smiles>

\begin{tabular}{lllll} 
& $\mathbf{1 a} \mathrm{R}=\mathrm{Me} ; \mathbf{1 b} \mathbf{R}=\mathrm{Et}$ & $\mathbf{2 a}$ & $\mathbf{4 a}$ \\
\hline Entry & catalyst & $\mathrm{R}$ & yield $(\%)^{b}$ & ee $(\%$ \\
\hline 1 & $\mathbf{3 a}$ & $\mathrm{Me}$ & 91 & 66 \\
2 & $\mathbf{3 b}$ & $\mathrm{Me}$ & 81 & 80 \\
3 & $\mathbf{3 b}$ & Et & 97 & 74
\end{tabular}




\begin{tabular}{lllll}
4 & $\mathbf{3 c}$ & $\mathrm{Me}$ & 22 & 54 \\
5 & $\mathbf{3 d}$ & $\mathrm{Me}$ & 47 & 64 \\
6 & $\mathbf{3 e}$ & $\mathrm{Me}$ & 13 & 54 \\
7 & $\mathbf{3 f}$ & $\mathrm{Me}$ & 23 & 68 \\
8 & $\mathbf{3 g}$ & $\mathrm{Me}$ & 15 & 17 \\
9 & $\mathbf{3 h}$ & $\mathrm{Me}$ & 30 & 8 \\
10 & $\mathbf{3 i}$ & $\mathrm{Me}$ & 10 & 32 \\
\hline
\end{tabular}

$\bar{a}$ To a mixture of catalyst 3a-i $(20 \mathrm{~mol} \%)$ and enamine $\mathbf{1}(0.3 \mathrm{mmol})$, in toluene:AcOEt 9:1 $(0.25 \mathrm{~mL})$, alkylidenmalononitrile $2(0.1 \mathrm{mmol})$ was added. ${ }^{b}$ Isolated yield after column chromatography $\left(\mathrm{SiO}_{2}, n\right.$-hexane:AcOEt 7:3). ${ }^{c}$ Determined by chiral HPLC analysis (Daicel Chiralpak IB, $n$-hexane:iPrOH 70:30, $1 \mathrm{~mL} / \mathrm{min}$ ).

Although promising results of enantioselectivities were obtained with catalysts $\mathbf{3 a - f}$, the best results in terms of both reactivity and enantioselectivity were achieved using bis-cinchona $\mathbf{3 b}$ (Table 1, entry 2). In addition, slight differences were observed for enamines 1a-b, with a methyl or an ethyl ester group, respectively (entries 2 and 3); while enamine 1a provided higher enantioselectivity (entry 2), enamine $\mathbf{1 b}$ gave better yield (entry 3 ). These results encouraged us to continue with both enamines 1a-b in the subsequent study, where different key parameters of this model reaction using catalyst $\mathbf{3 b}$ were tested (Table 2).

Table 2. Screening of the reaction conditions for the synthesis of chiral 1,4-DHPs 4aa-ba. ${ }^{a}$

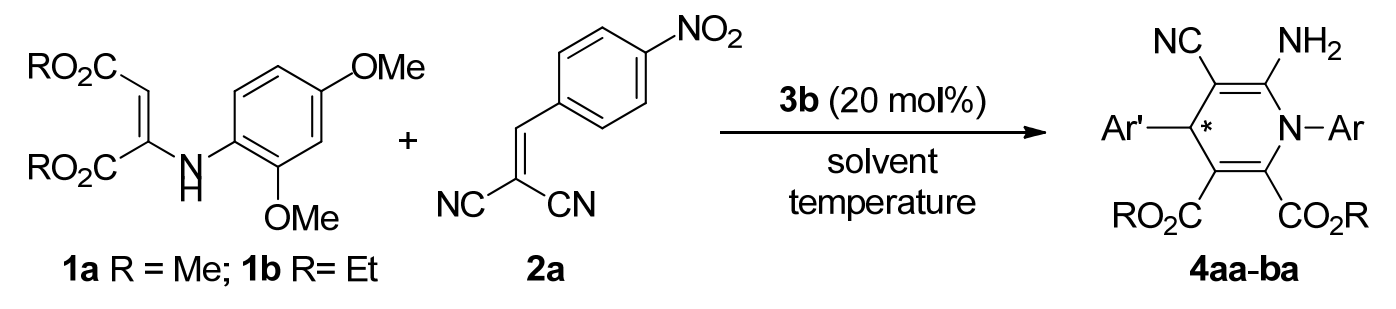

\begin{tabular}{llllllc}
\hline Entry & solvent $(\mathrm{mL})$ & $\begin{array}{l}\text { enamine } \\
\text { (equiv.) }\end{array}$ & $\mathrm{T}\left({ }^{\circ} \mathrm{C}\right)$ & time $(\mathrm{h})$ & yield $(\%)^{b}$ & ee $(\%)^{c}$ \\
\hline 1 & AcOEt $(0.25)$ & $\mathbf{1 a}(3)$ & 10 & 72 & 89 & 64 \\
2 & Toluene:AcOEt 9:1 (0.25) & $\mathbf{1 a}(3)$ & 10 & 72 & 81 & 80
\end{tabular}




$\begin{array}{lllllll}3 & \text { Toluene:AcOEt 95:5 (0.25) } & \mathbf{1 a}(3) & 10 & 72 & 70 & 80 \\ 4 & \text { Toluene }(0.25) & \mathbf{1 a}(3) & 10 & 72 & 88 & 82 \\ 5 & \text { Toluene:AcOEt 9:1 (0.25) } & \mathbf{1 a}(3) & 0 & 72 & 32 & 80 \\ 6 & \text { Toluene:AcOEt 9:1 (0.25) } & \mathbf{1 a}(3) & -18 & 120 & <5 & 85 \\ 7 & \text { Toluene:AcOEt 9:1 (0.5) } & \mathbf{1 b}(2) & 10 & 120 & 49 & 68 \\ 8 & \text { Toluene:AcOEt 9:1 (0.5) } & \mathbf{1 b}(3) & 10 & 72 & 93 & 76 \\ 9 & \text { Toluene:AcOEt 9:1 (0.5) } & \mathbf{1 b}(4) & 10 & 72 & 93 & 76 \\ 10 & \text { Toluene:AcOEt 9:1 (0.5) } & \mathbf{1 b}(5) & 10 & 72 & 95 & 76 \\ 11 & \text { Toluene:AcOEt 9:1 (0.25) } & \mathbf{1 b}(3) & 10 & 72 & 97 & 76 \\ 12 & \text { Toluene:AcOEt 9:1 (0.25) } & \mathbf{1 b}(3) & -18 & 72 & <5 & 90\end{array}$

\footnotetext{
${ }^{a}$ To a mixture of catalyst $\mathbf{3 b}(20 \mathrm{~mol} \%, 18.17 \mathrm{mg})$ and enamine $\mathbf{1}(0.3-0.5 \mathrm{mmol})$, in the corresponding solvent $(0.25-0.5 \mathrm{~mL})$, alkylidenmalononitrile $2(0.1 \mathrm{mmol})$ was added. ${ }^{b}$ Isolated yield after column chromatography $\left(\mathrm{SiO}_{2}, n\right.$-hexane:AcOEt 7:3). ${ }^{c}$ Determined by chiral HPLC analysis (Daicel Chiralpak IB, $n$-hexane: $i$ PrOH 70:30, $1 \mathrm{~mL} / \mathrm{min}$ ).
}

The ratio toluene:AcOEt was examined to find the best polarity for the process (Table 2, entries 1-4). Although in this case, toluene was the best solvent, in further experiments the use of $10 \%$ of AcOEt was crucial to obtain good yields without compromising the enantioselectivity. Consequently, both media, toluene:AcOEt 9:1 and only toluene, were used to develop the final scope since better results were observed in one of the options, depending on the substrates (Scheme 2). Decreasing the temperature to $-18^{\circ} \mathrm{C}$ led to a slightly improved enantioselectivity but unfortunately, with a very low reactivity (entries 5, 6 and 12). The ratio of enamine:malononitrile was also explored, but no remarkable differences were found with more than three equivalents of $\mathbf{1}$ (compare entries 8-10). In contrast, slower reaction rate and poorer enantioselectivity were found when only 2 equivalents of $\mathbf{1 b}$ were used (entry 7 ). The concentration of the reaction had no significant effect on the enantioselectivity or the 
reactivity of the process (compare entries 8 and 11). Therefore, the best reaction conditions were found to be $0.25 \mathrm{~mL}$ of toluene:AcOEt 9:1 or only toluene, $20 \mathrm{~mol} \%$ of catalyst $\mathbf{3 b}$ and 3 equivalents of enamine 1 , at $10{ }^{\circ} \mathrm{C}$. In order to develop the methodology, the efficiency of these conditions was further explored for a variety of enamines 1a-g and alkylidenmalononitriles 2a-j (Scheme 2).

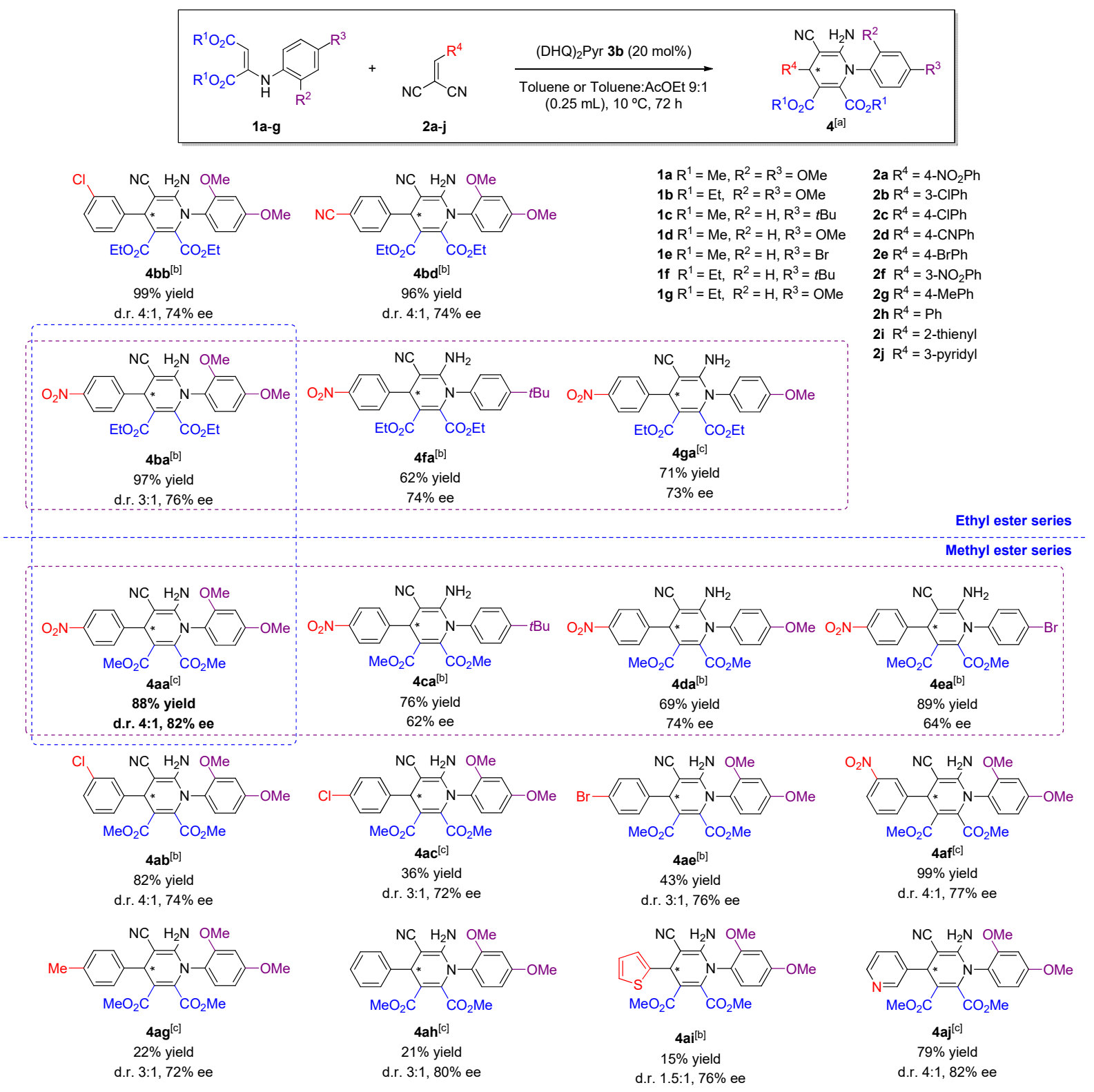

Scheme 2. Scope of the organocatalytic synthesis of enantiomerically enriched 1,4-DHPs 4.

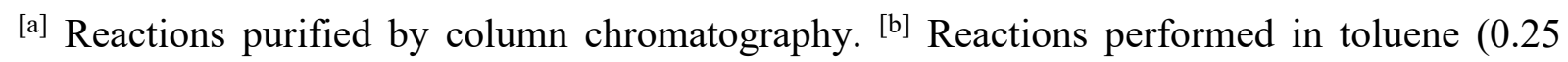
$\mathrm{mL}){ }^{[\mathrm{c}]}$ Reactions performed in toluene:AcOEt 9:1 $(0.25 \mathrm{~mL})$. 
In general, the resulting chiral 1,4-DHPs 4 were achieved with good enantioselectivities (up to $82 \%$ ) and moderate to very good yield (up to $99 \%$ ). While the correlation between the electronic environment of the enamines $\mathbf{1}$ and the reactivity and the enantioselectivity of the process remains unclear, it seems that better results were obtained with enamines possessing two $\mathrm{MeO}$ groups in the aniline ring (i.e., 1a and $\mathbf{1 b}$ ), in comparison with the differently substituted $p$ - $t \mathrm{Bu}, p$-OMe, $p$-Br-enamines [see: 4aa $v s$ 4ca-ea (methyl ester enamine series); and 4ba vs 4fa-ga (ethyl ester enamine series)]. As previously shown in Tables 1 and 2 for enamines 1a-b, slight differences were observed for methyl ester and ethyl ester substituted enamines (see: 4aa $v s$ 4ba; 4ca $v s$ 4fa; 4da $v s$ 4ga; 4ab vs 4bb). Regarding the malononitriles 2, although the results do not suggest a clear dependence of the reactivity of the process on the electronic properties of the starting malononitriles, those synthesized from deactivated aldehydes showed a clear reduced reactivity (see 4ag-ai). Moreover, it seems that metasubstitution of the aromatic ring of the starting aldehyde provides in general, better yields in comparison with the analogous para-substituted substrates (see, 4af $v s$ 4aa and 4ab vs 4ac). The structural complexity and the high functionalization of the final products of this protocol have been confirmed by the single-crystal analysis of compounds 4aa and 4ae (Figure S53S55).

Based on the literature data for the non-asymmetric version of this reaction, ${ }^{10}$ and our previous work, ${ }^{8 \mathrm{f}}$ we propose the mechanism depicted in Scheme 3. 


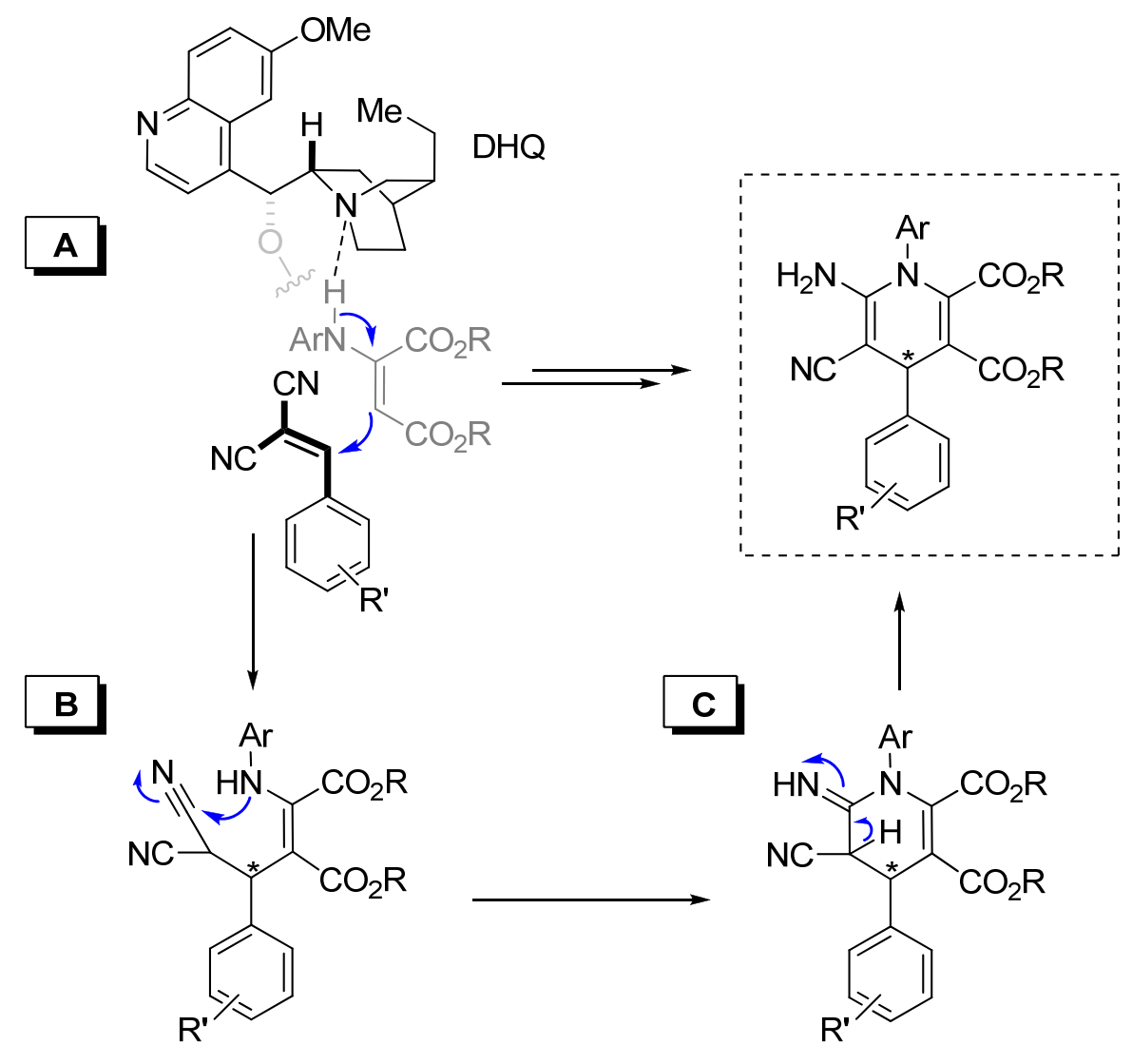

Scheme 3. Plausible reaction mechanism.

We think that the catalyst mainly participates in the first step of the reaction (Scheme 3, A), since $\mathrm{Et}_{3} \mathrm{~N}$ is able to promote this reaction in the racemic version. ${ }^{10}$ Thus, the Michael reaction between the enamine and the malononitrile would be promoted by the basic catalyst, driving the addition of the enamine to the Michael acceptor in an enantioselective manner. The ring is then formed through an intramolecular nucleophilic addition of the NH to a nitrile group, followed by an imine-enamine tautomerization (Scheme 3, B and C, respectively). A plausible proposal about the absolute configuration of the final products $\mathbf{4}$ has been provided in the Supporting Information (Figure S56). 


\section{Conclusions}

In summary, we have reported an unprecedented approach for the enantioselective formation of 1,4-dihydropyridine derivatives 4 under mild conditions and with operational simplicity. Using bis-cinchona $\mathbf{3 b}$ as a catalyst, the final adducts were reached with very good results. A broad structural variety of 1,4-dihydropyridine derivatives is successfully achieved with our developed procedure. This work is one of the scarce asymmetric catalytic examples reported for the synthesis of enantiomerically enriched 1,4-dihydropyridines. Better understanding of the mechanism and studies into the applicability of the final compounds are ongoing in our laboratories.

\section{Experimental Section}

\section{General experimental methods and instrumentation}

Purification of reaction products was carried out by flash chromatography using silica gel (0.063-0.200 mm). Analytical thin layer chromatography was performed on $0.25 \mathrm{~mm}$ silica gel 60-F plates. ESI ionization method and mass analyzer type MicroTof-Q were used for the HRMS measurements. ${ }^{1} \mathrm{H}-\mathrm{NMR}$ spectra were recorded at 300 and $400 \mathrm{MHz} ;{ }^{13} \mathrm{C}-\mathrm{APT}-\mathrm{NMR}$ spectra were recorded at $75 \mathrm{MHz} ; \mathrm{CDCl}_{3}$ and DMSO- $d_{6}$ as the solvents. Chemical shifts were reported in the $\delta$ scale relative to residual $\mathrm{CHCl}_{3}(7.26 \mathrm{ppm})$ and $\mathrm{DMSO}(2.50 \mathrm{ppm})$ for ${ }^{1} \mathrm{H}$ $\mathrm{NMR}$ and to the central line of $\mathrm{CDCl}_{3}(77 \mathrm{ppm})$ and $\mathrm{DMSO}-d_{6}(39.43 \mathrm{ppm})$ for ${ }^{13} \mathrm{C}$ APT-NMR.

All commercially available solvents and reagents were used as received.

Materials. Spectral data for enamines $1 \mathbf{b},{ }^{8 \mathrm{f}} \mathbf{1 c},{ }^{11} \mathbf{1 d},{ }^{12} \mathbf{1 e},{ }^{12} \mathbf{1 f},{ }^{8 \mathrm{f}} \mathbf{1 g},{ }^{8 \mathrm{f}}$ and malononitriles $\mathbf{2 a},{ }^{13}$ $\mathbf{2 b},{ }^{14} \mathbf{2 c},{ }^{13} \mathbf{2 d},{ }^{15} \mathbf{2 e},{ }^{16} \mathbf{2 f},{ }^{14} \mathbf{2 g},{ }^{14} \mathbf{2 h},{ }^{13} \mathbf{2} \mathbf{i},{ }^{14} \mathbf{2 j},{ }^{17}$ are consistent with values previously reported in the literature. For the spectra and HPLC chromatograms of products 4, see supporting information. 
Dimethyl 2-(2,4-dimethoxyphenylamino)maleate (1a): Following our previous developed procedure, ${ }^{8 \mathrm{f}}$ compound 1a was obtained as a yellow oil in a $55 \%$ yield. ${ }^{1} \mathrm{H}-\mathrm{NMR}(300 \mathrm{MHz}$, $\left.\mathrm{CDCl}_{3}\right) \delta 3.69(\mathrm{~s}, 3 \mathrm{H}), 3.72(\mathrm{~s}, 3 \mathrm{H}), 3.77(\mathrm{~s}, 3 \mathrm{H}), 3.79(\mathrm{~s}, 3 \mathrm{H}), 5.30(\mathrm{~s}, 1 \mathrm{H}), 6.38(\mathrm{dd}, J=8.6$ $\mathrm{Hz}, J=2.6 \mathrm{~Hz}, 1 \mathrm{H}), 6.46(\mathrm{~d}, J=2.6 \mathrm{~Hz}, 1 \mathrm{H}), 6.76(\mathrm{~d}, J=8.7 \mathrm{~Hz}, 1 \mathrm{H}), 9.52$ (br s, $1 \mathrm{H}) .{ }^{13} \mathrm{C}-$ APT-NMR (75 MHz, $\left.\mathrm{CDCl}_{3}\right) \delta 50.9$ (1C), 52.4 (1C), 55.3 (1C), 55.4 (1C), 90.5 (1C), 99.1 (1C), 103.7 (1C), 121.8 (1C), 122.6 (1C), 148.6 (1C), 152.1 (1C), 157.4 (1C), 164.5 (1C), 170.0 (1C). IR (neat) $\left(\mathrm{cm}^{-1}\right) \vee 3294,3007,2983,2951,2930,2832,1741,1673,1604,1588$, $1518,1463,1450,1435,1413,1392,1331,1278,1203,1147,1121,1036,1027,977,828$, 798, 775, 635. HRMS (ESI+) calcd for $\mathrm{C}_{14} \mathrm{H}_{17} \mathrm{NNaO}_{6} 318.0948$; found 318.0947 [M+Na].

\section{General procedure for the synthesis of 1,4-dihydropyridines 4}

To a mixture of catalyst $\mathbf{3 b}(20 \mathrm{~mol} \%, 18.17 \mathrm{mg})$ and enamine $\mathbf{1}(0.3 \mathrm{mmol})$, in toluene:AcOEt 9:1 or toluene $(0.25 \mathrm{~mL})$, alkylidenmalononitrile $2(0.1 \mathrm{mmol})$ was added. The reaction mixture was stirred 3 days at $10^{\circ} \mathrm{C}$. Then, the solvent was evaporated under vacuum, and the reaction crude was purified by column chromatography $\left(\mathrm{SiO}_{2}, n\right.$-hexane:AcOEt 7:3 to 1:1), giving rise to the corresponding final chiral adduct 4 (Scheme 2).

\section{Dimethyl}

6-amino-5-cyano-1-(2,4-dimethoxyphenyl)-4-(4-nitrophenyl)-1,4-

\section{dihydropyridine-2,3-dicarboxylate (4aa)}

Following the general procedure, compound 4aa was obtained after $72 \mathrm{~h}$ of reaction at $10{ }^{\circ} \mathrm{C}$ and was purified by column chromatography ( $n$-hexane:AcOEt 7:3 to $1: 1$ ), as a yellow solid in $88 \%$ yield $\left(43.5 \mathrm{mg}\right.$ ). M.p. $>98{ }^{\circ} \mathrm{C}$ decomp. The ee of the product was determined to be $82 \%$ by HPLC using a Daicel Chiralpak IB column $(n$-hexane $/ i$-PrOH $=70: 30$, flow rate 1

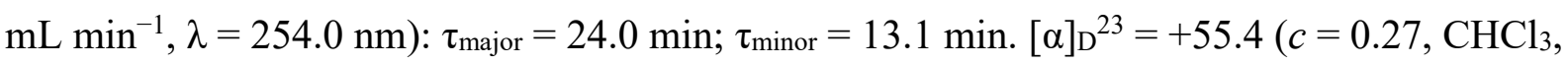
$82 \%$ ee). ${ }^{1} \mathrm{H}-\mathrm{NMR}\left(300 \mathrm{MHz}, \mathrm{DMSO}-d_{6}\right) \delta 3.33(\mathrm{~s}, 0.6 \mathrm{H}), 3.37$ (s, 2.4H), $3.48(\mathrm{~s}, 0.6 \mathrm{H}), 3.49$ 
(s, 2.4H), $3.82(\mathrm{~s}, 3.6 \mathrm{H}), 3.90(\mathrm{~s}, 2.4 \mathrm{H}), 4.62(\mathrm{~s}, 0.8 \mathrm{H}), 4.65(\mathrm{~s}, 0.2 \mathrm{H}), 5.70(\mathrm{br} \mathrm{s}, 2 \mathrm{H}), 6.54-$ $6.57(\mathrm{~m}, 0.8 \mathrm{H}), 6.57-6.59(\mathrm{~m}, 0.2 \mathrm{H}), 6.69(\mathrm{~d}, J=2.6 \mathrm{~Hz}, 0.2 \mathrm{H}), 6.72(\mathrm{~d}, J=2.6 \mathrm{~Hz}, 0.8 \mathrm{H})$, $7.14(\mathrm{~d}, J=8.7 \mathrm{~Hz}, 0.8 \mathrm{H}), 7.24(\mathrm{~d}, J=8.7 \mathrm{~Hz}, 0.2 \mathrm{H}), 7.53-7.59(\mathrm{~m}, 0.4 \mathrm{H}), 7.64-7.70(\mathrm{~m}$, 1.6H), 8.25-8.30 (m, 0.4H), 8.29-8.34 (m, 1.6H). ${ }^{13} \mathrm{C}-\mathrm{APT}-\mathrm{NMR}\left(75 \mathrm{MHz}, \mathrm{DMSO}-d_{6}\right) \delta 39.0$ (1C), 51.8 (1C), 52.3 (1C), 55.5 (1C), 56.2 (1C), 58.1 (1C), 99.1 (1C), 102.7 (1C), 105.0 (1C), 115.7 (1C), 120.6 (1C), 123.8 (2C), 128.2 (2C), 132.2 (1C), 143.4 (1C), 146.4 (1C), 151.2 (1C), $153.1(1 \mathrm{C}), 157.7(1 \mathrm{C}), 161.8(1 \mathrm{C}), 162.6(1 \mathrm{C}), 164.6(1 \mathrm{C}) . \mathrm{IR}$ (neat) $\left(\mathrm{cm}^{-1}\right) \vee 3452$, $3345,3000,2948,2841,2180,1743,1705,1646,1610,1568,1508,1486,1454,1435,1415$, $1354,1335,1312,1285,1261,1208,1163,1110,1054,1027,1009,973,933,865,834,804$, 778, 764, 730. HRMS (ESI+) calcd for $\mathrm{C}_{24} \mathrm{H}_{22} \mathrm{~N}_{4} \mathrm{NaO}_{8} 517.1330$; found 517.1302 [M+Na].

\section{Diethyl}

6-amino-5-cyano-1-(2,4-dimethoxyphenyl)-4-(4-nitrophenyl)-1,4-

\section{dihydropyridine-2,3-dicarboxylate (4ba)}

Following the general procedure, compound $4 \mathbf{b a}$ was obtained after $72 \mathrm{~h}$ of reaction at $10{ }^{\circ} \mathrm{C}$ and was purified by column chromatography ( $n$-hexane:AcOEt 7:3 to 1:1), as a yellow solid in $97 \%$ yield $(50.7 \mathrm{mg})$. M.p. $124-126^{\circ} \mathrm{C}$. The ee of the product was determined to be $76 \%$ by HPLC using a Daicel Chiralpak IB column $\left(n\right.$-hexane $/ i$-PrOH $=80: 20$, flow rate $1 \mathrm{~mL} \mathrm{~min}{ }^{-1}$, $\lambda=276.0 \mathrm{~nm}): \tau_{\text {major }}=27.2 \mathrm{~min} ; \tau_{\text {minor }}=16.5 \mathrm{~min} .[\alpha]_{\mathrm{D}}{ }^{22}=+52.5\left(c=0.26, \mathrm{CHCl}_{3}, 76 \%\right.$ ee $)$. ${ }^{1} \mathrm{H}-\mathrm{NMR}\left(300 \mathrm{MHz}, \mathrm{DMSO}-d_{6}\right) \delta 0.88(\mathrm{t}, J=7.2 \mathrm{~Hz}, 0.75 \mathrm{H}), 0.89(\mathrm{t}, J=7.2 \mathrm{~Hz}, 2.25 \mathrm{H})$, $1.02(\mathrm{t}, J=7.1 \mathrm{~Hz}, 0.75 \mathrm{H}), 1.03(\mathrm{t}, J=7.1 \mathrm{~Hz}, 2.25 \mathrm{H}), 3.81(\mathrm{~s}, 3.75 \mathrm{H}), 3.72-3.97(\mathrm{~m}, 4 \mathrm{H})$, $3.91(\mathrm{~s}, 2.25 \mathrm{H}), 4.62(\mathrm{~s}, 0.75 \mathrm{H}), 4.65(\mathrm{~s}, 0.25 \mathrm{H}), 5.68(\mathrm{br} \mathrm{s}, 2 \mathrm{H}), 6.54-6.58(\mathrm{~m}, 0.75 \mathrm{H}), 6.58-$ $6.62(\mathrm{~m}, 0.25 \mathrm{H}), 6.68(\mathrm{~d}, J=2.5 \mathrm{~Hz}, 0.25 \mathrm{H}), 6.71(\mathrm{~d}, J=2.1 \mathrm{~Hz}, 0.75 \mathrm{H}), 7.14(\mathrm{~d}, J=8.7 \mathrm{~Hz}$, $0.75 \mathrm{H}), 7.25(\mathrm{~d}, J=8.7 \mathrm{~Hz}, 0.25 \mathrm{H}), 7.55-7.60(\mathrm{~m}, 0.5 \mathrm{H}), 7.65-7.72(\mathrm{~m}, 1.5 \mathrm{H}), 8.24-8.30(\mathrm{~m}$, 0.5H), 8.29-8.35 (m, 1.5H). ${ }^{13} \mathrm{C}-\mathrm{APT}-\mathrm{NMR}\left(75 \mathrm{MHz}, \mathrm{DMSO}-d_{6}\right) \delta 13.2(1 \mathrm{C}), 13.6$ (1C), 39.1 (1C), 55.6 (1C), 56.2 (1C), 58.0 (1C), 60.3 (1C), 61.2 (1C), 99.1 (1C), 102.7 (1C), 105.0 (1C), 
115.7 (1C), 120.7 (1C), 123.7 (2C), 128.4 (2C), 132.5 (1C), 143.2 (1C), 146.4 (1C), 151.3 (1C), 153.3 (1C), 157.8 (1C), 161.9 (1C), 162.0 (1C), 164.1 (1C). IR (neat) $\left(\mathrm{cm}^{-1}\right) \vee 3461$, $3346,3185,2983,2937,2842,2181,1738,1700,1646,1605,1568,1508,1463,1418,1391$, $1369,1343,1309,1285,1260,1206,1162,1105,1054,1024,935,922,858,821,729,708$. HRMS (ESI+) calcd for $\mathrm{C}_{26} \mathrm{H}_{25} \mathrm{~N}_{4} \mathrm{O}_{8} 521.1667$; found 521.1645 [M-H].

\section{Dimethyl 6-amino-1-(4-tert-butylphenyl)-5-cyano-4-(4-nitrophenyl)-1,4-dihydropyridine- 2,3-dicarboxylate (4ca)}

Following the general procedure, compound $4 \mathbf{c a}$ was obtained after $72 \mathrm{~h}$ of reaction at $10^{\circ} \mathrm{C}$ and was purified by column chromatography ( $n$-hexane:AcOEt 7:3 to $1: 1$ ), as a yellow solid in $76 \%$ yield $(37.3 \mathrm{mg})$. M.p. $>104{ }^{\circ} \mathrm{C}$ decomp. The ee of the product was determined to be $62 \%$ by HPLC using a Daicel Chiralpak IB column $(n$-hexane $/ i$-PrOH $=70: 30$, flow rate 1 $\left.\mathrm{mL} \min ^{-1}, \lambda=238.0 \mathrm{~nm}\right): \tau_{\text {major }}=10.0 \mathrm{~min} ; \tau_{\text {minor }}=7.7 \mathrm{~min} .[\alpha]_{\mathrm{D}^{27}}=+75.3\left(c=0.24, \mathrm{CHCl}_{3}\right.$, $62 \%$ ee). ${ }^{1} \mathrm{H}-\mathrm{NMR}\left(300 \mathrm{MHz}, \mathrm{DMSO}-d_{6}\right) \delta 1.31(\mathrm{~s}, 9 \mathrm{H}), 3.31(\mathrm{~s}, 3 \mathrm{H}), 3.52(\mathrm{~s}, 3 \mathrm{H}), 4.67(\mathrm{~s}$, 1H), $5.75($ br s, 2H), $7.26(\mathrm{~d}, J=8.6 \mathrm{~Hz}, 2 \mathrm{H}), 7.52(\mathrm{~d}, J=8.6 \mathrm{~Hz}, 2 \mathrm{H}), 7.58(\mathrm{~d}, J=8.8 \mathrm{~Hz}$, 2H), $8.30(\mathrm{~d}, J=8.8 \mathrm{~Hz}, 2 \mathrm{H}) .{ }^{13} \mathrm{C}-\mathrm{APT}-\mathrm{NMR}\left(75 \mathrm{MHz}, \mathrm{DMSO}-d_{6}\right) \delta 30.8(3 \mathrm{C}), 34.5(1 \mathrm{C})$, 38.6 (1C), 51.9 (1C), 52.2 (1C), 58.5 (1C), 103.2 (1C), 109.4 (1C), 120.5 (1C), 124.2 (2C), 126.2 (2C), 127.8 (2C), 129.6 (2C), 132.3 (1C), 142.7 (1C), 146.4 (1C), 151.1 (1C), 152.5 (1C), 162.6 (1C), 164.6 (1C). IR (neat) $\left(\mathrm{cm}^{-1}\right) \vee 3471,3357,2953,2850,2183,1746,1708$, $1648,1605,1569,1519,1461,1417,1344,1222,1108,1054,1014,973,931,875,860,819$, 728. HRMS (ESI+) calcd for $\mathrm{C}_{26} \mathrm{H}_{27} \mathrm{~N}_{4} \mathrm{O}_{6} 491.1925$; found 491.1898 [M+H].

Dimethyl 6-amino-5-cyano-1-(4-methoxyphenyl)-4-(4-nitrophenyl)-1,4-dihydropyridine2,3-dicarboxylate (4da) 
Following the general procedure, compound 4 da was obtained after $72 \mathrm{~h}$ of reaction at $10{ }^{\circ} \mathrm{C}$ and was purified by column chromatography ( $n$-hexane:AcOEt $7: 3$ to $1: 1$ ), as a yellow solid in $69 \%$ yield ( $32 \mathrm{mg}$ ). M.p. $153-155^{\circ} \mathrm{C}$. The ee of the product was determined to be $74 \%$ by HPLC using a Daicel Chiralpak IB column $\left(n\right.$-hexane $/ i$-PrOH $=70: 30$, flow rate $1 \mathrm{~mL} \mathrm{~min}{ }^{-1}$, $\lambda=238.0 \mathrm{~nm}): \tau_{\text {major }}=20.1 \mathrm{~min} ; \tau_{\text {minor }}=14.3 \mathrm{~min} .[\alpha]_{\mathrm{D}}{ }^{26}=+34.5\left(c=0.26, \mathrm{CHCl}_{3}, 74 \%\right.$ ee $)$. ${ }^{1} \mathrm{H}-\mathrm{NMR}(300 \mathrm{MHz}, \mathrm{DMSO}-d 6) \delta 3.38(\mathrm{~s}, 3 \mathrm{H}), 3.52(\mathrm{~s}, 3 \mathrm{H}), 3.81(\mathrm{~s}, 3 \mathrm{H}), 4.66(\mathrm{~s}, 1 \mathrm{H}), 5.76$ (br s, 2H), $7.03(\mathrm{~d}, J=9.0 \mathrm{~Hz}, 2 \mathrm{H}), 7.28(\mathrm{~d}, J=8.9 \mathrm{~Hz}, 2 \mathrm{H}), 7.58(\mathrm{~d}, J=8.8 \mathrm{~Hz}, 2 \mathrm{H}), 8.29(\mathrm{~d}$, $J=8.8 \mathrm{~Hz}, 2 \mathrm{H}) .{ }^{13} \mathrm{C}-\mathrm{APT}-\mathrm{NMR}\left(75 \mathrm{MHz}, \mathrm{DMSO}-d_{6}\right) \delta 38.6(1 \mathrm{C}), 51.9(1 \mathrm{C}), 52.3(1 \mathrm{C}), 55.4$ (1C), 58.1 (1C), 102.8 (1C), 114.6 (2C), 120.5 (1C), 124.1 (2C), 127.1 (1C), 127.9 (2C), 131.4 (2C), 142.9 (1C), 146.4 (1C), 151.3 (1C), 152.7 (1C), 159.9 (1C), 162.6 (1C), 164.6 (1C). IR (neat) $\left(\mathrm{cm}^{-1}\right) \vee 3458,3354,3215,2954,2841,2181,2120,1742,1707,1648,1605$, $1567,1508,1457,1432,1414,1345,1302,1246,1221,1181,1106,1056,1024,972,930$, 874, 860, 820, 806, 747, 728, 713. HRMS (ESI+) calcd for $\mathrm{C}_{23} \mathrm{H}_{21} \mathrm{~N}_{4} \mathrm{O}_{7}$ 465.1405; found $465.1391[\mathrm{M}+\mathrm{H}]$.

\section{Dimethyl 6-amino-1-(4-bromophenyl)-5-cyano-4-(4-nitrophenyl)-1,4-dihydropyridine-}

\section{2,3-dicarboxylate (4ea)}

Following the general procedure, compound 4 ea was obtained after $72 \mathrm{~h}$ of reaction at $10{ }^{\circ} \mathrm{C}$ and was purified by column chromatography ( $n$-hexane:AcOEt $7: 3$ to $1: 1$ ), as a yellow solid in $89 \%$ yield $\left(45.7 \mathrm{mg}\right.$ ). M.p. $>80{ }^{\circ} \mathrm{C}$ decomp. The ee of the product was determined to be 64\% by HPLC using a Daicel Chiralpak IB column $(n$-hexane $/ i$-PrOH $=70: 30$, flow rate 1 $\left.\mathrm{mL} \min ^{-1}, \lambda=238.0 \mathrm{~nm}\right): \tau_{\text {major }}=17.0 \mathrm{~min} ; \tau_{\text {minor }}=13.4 \mathrm{~min} .[\alpha]_{\mathrm{D}}{ }^{27}=-4.3\left(c=0.20, \mathrm{CHCl}_{3}\right.$, 64\% ee). ${ }^{1} \mathrm{H}-\mathrm{NMR}(300 \mathrm{MHz}, \mathrm{DMSO}-d 6) \delta 3.40$ (s, 3H), 3.52 (s, 3H), 4.67 (s, 1H), 5.94 (br s, 2H), $7.32(\mathrm{~d}, J=8.7 \mathrm{~Hz}, 2 \mathrm{H}), 7.58(\mathrm{~d}, J=8.8 \mathrm{~Hz}, 2 \mathrm{H}), 7.71(\mathrm{~d}, J=8.7 \mathrm{~Hz}, 2 \mathrm{H}), 8.29(\mathrm{~d}, J=$ $8.8 \mathrm{~Hz}, 2 \mathrm{H}) .{ }^{13} \mathrm{C}-\mathrm{APT}-\mathrm{NMR}\left(75 \mathrm{MHz}, \mathrm{DMSO}-d_{6}\right) \delta 38.7$ (1C), 52.0 (1C), 52.5 (1C), 58.4 
(1C), 103.5 (1C), 120.4 (1C), 123.5 (1C), 124.2 (2C), 128.0 (2C), 132.4 (2C), 132.6 (2C), 134.4 (1C), 142.2 (1C), 146.5 (1C), 150.8 (1C), 152.5 (1C), 162.6 (1C), 164.6 (1C). IR (neat) $\left(\mathrm{cm}^{-1}\right) \vee 3336,3110,2955,2918,2849,2217,1731,1592,1560,1521,1488,1436,1398$ 1346, 1240, 1160, 1106, 1071, 1010, 975, 851, 823, 785, 741, 720. HRMS (ESI+) calcd for $\mathrm{C}_{22} \mathrm{H}_{18} \mathrm{BrN}_{4} \mathrm{O}_{6}$ 513.0404; found 513.0364 [M+H].

\section{Diethyl 6-amino-1-(4-tert-butylphenyl)-5-cyano-4-(4-nitrophenyl)-1,4-dihydropyridine-}

\section{2,3-dicarboxylate (4fa)}

Following the general procedure, compound $\mathbf{4 f a}$ was obtained after $72 \mathrm{~h}$ of reaction at $10^{\circ} \mathrm{C}$ and was purified by column chromatography ( $n$-hexane:AcOEt 7:3 to $1: 1$ ), as a yellow solid in $62 \%$ yield $(32.2 \mathrm{mg})$. M.p. $137-140^{\circ} \mathrm{C}$. The ee of the product was determined to be $74 \%$ by HPLC using a Daicel Chiralpak IB column ( $n$-hexane $/ i-\mathrm{PrOH}=80: 20$, flow rate $1 \mathrm{~mL} \mathrm{~min}{ }^{-1}$, $\lambda=254.0 \mathrm{~nm}): \tau_{\text {major }}=11.1 \mathrm{~min} ; \tau_{\text {minor }}=8.1 \mathrm{~min} .[\alpha]_{\mathrm{D}^{27}}=+64.5\left(c=0.24, \mathrm{CHCl}_{3}, 74 \%\right.$ ee $)$. ${ }^{1} \mathrm{H}-\mathrm{NMR}(300 \mathrm{MHz}, \mathrm{DMSO}-d 6) \delta 0.72(\mathrm{t}, J=7.1 \mathrm{~Hz}, 3 \mathrm{H}), 1.03(\mathrm{t}, J=7.1,3 \mathrm{H}), 1.3(\mathrm{~s}, 9 \mathrm{H})$, 3.67-3.88 (m, 2H), $3.96(\mathrm{q}, J=7.1 \mathrm{~Hz}, 2 \mathrm{H}), 4.68(\mathrm{~s}, 1 \mathrm{H}), 5.73($ br s, 2H), $7.28(\mathrm{~d}, J=8.6 \mathrm{~Hz}$, 2H), $7.52(\mathrm{~d}, J=8.7 \mathrm{~Hz}, 2 \mathrm{H}), 7.59(\mathrm{~d}, J=8.8 \mathrm{~Hz}, 2 \mathrm{H}), 8.30(\mathrm{~d}, J=8.8 \mathrm{~Hz}, 2 \mathrm{H}) .{ }^{13} \mathrm{C}-\mathrm{APT}-$ NMR (75 MHz, DMSO-d6) $\delta 12.9$ (1C), 13.6 (1C), 30.8 (3C), 34.5 (1C), 38.8 (1C), 58.3 (1C), 60.5 (1C), 61.3 (1C), 103.2 (1C), 120.5 (1C), 124.1 (2C), 126.2 (2C), $128.1(2 \mathrm{C}), 129.9$ (2C), 132.3 (1C), 142.5 (1C), 146.4 (1C), 151.1 (1C), 152.6 (1C), 152.7 (1C), 162.0 (1C), 164.1 (1C). IR (neat) $\left(\mathrm{cm}^{-1}\right) \vee 3476,3313,3213,2961,2937,2905,2866,2185,1741,1699,1650$, $1595,1569,1520,1504,1477,1464,1447,1392,1369,1344,1269,1244,1217,1200,1173$, $1149,1103,1057,1034,1011,956,905,855,843,818,743,728$. HRMS (ESI+) calcd for $\mathrm{C}_{28} \mathrm{H}_{31} \mathrm{~N}_{4} \mathrm{O}_{6} 519.2238$; found $519.2235[\mathrm{M}+\mathrm{H}]$. 


\section{Diethyl 6-amino-5-cyano-1-(4-methoxyphenyl)-4-(4-nitrophenyl)-1,4-dihydropyridine-}

\section{2,3-dicarboxylate (4ga)}

Following the general procedure, compound 4 ga was obtained after $72 \mathrm{~h}$ of reaction at $10{ }^{\circ} \mathrm{C}$ and was purified by column chromatography ( $n$-hexane:AcOEt 7:3 to 1:1), as a yellow solid in $71 \%$ yield $(35.0 \mathrm{mg})$. M.p. $138-141^{\circ} \mathrm{C}$. The ee of the product was determined to be $73 \%$ by HPLC using a Daicel Chiralpak IB column $\left(n\right.$-hexane $/ i$-PrOH $=80: 20$, flow rate $1 \mathrm{~mL} \mathrm{~min}{ }^{-1}$, $\lambda=236.4 \mathrm{~nm}): \tau_{\text {major }}=29.4 \mathrm{~min} ; \tau_{\text {minor }}=20.2 \mathrm{~min} .[\alpha]_{\mathrm{D}}{ }^{22}=+43.0\left(c=0.26, \mathrm{CHCl}_{3}, 60 \%\right) .{ }^{1} \mathrm{H}-$ NMR (300 MHz, DMSO-d6) $\delta 0.89(\mathrm{t}, J=7.1 \mathrm{~Hz}, 3 \mathrm{H}), 1.03(\mathrm{t}, J=7.1 \mathrm{~Hz}, 3 \mathrm{H}), 3.74-3.99$ (m, 4H), $3.80(\mathrm{~s}, 3 \mathrm{H}), 4.67(\mathrm{~s}, 1 \mathrm{H}), 5.74(\mathrm{br} \mathrm{s}, 2 \mathrm{H}), 7.03(\mathrm{~d}, J=9.0 \mathrm{~Hz}, 2 \mathrm{H}), 7.29(\mathrm{~d}, J=8.9$ $\mathrm{Hz}, 2 \mathrm{H}), 7.58(\mathrm{~d}, J=8.8 \mathrm{~Hz}, 2 \mathrm{H}), 8.29(\mathrm{~d}, J=8.8 \mathrm{~Hz}, 2 \mathrm{H}) .{ }^{13} \mathrm{C}$-APT-NMR (75 MHz, DMSOd6) $\delta 13.1(1 \mathrm{C}), 13.6$ (1C), 38.8 (1C), 55.5 (1C), 58.0 (1C), 60.5 (1C), 61.4 (1C), 102.9 (1C), 114.6 (2C), 120.6 (1C), 124.1 (2C), 127.2 (1C), 128.1 (2C), 131.7 (2C), 142.7 (1C), 146.4 (1C), 151.3 (1C), 152.9 (1C), 160.1 (1C), 162.0 (1C), 164.1 (1C). IR (neat) $\left(\mathrm{cm}^{-1}\right) \vee 3452$, $3314,3214,3076,2958,2919,2850,2178,1741,1706,1693,1648,1596,1568,1522,1506$, $1462,1424,1390,1370,1345,1298,1253,1217,1200,1170,1151,1104,1058,1011,963$, 907, 852, 821, 784, 763, 746, 728. HRMS (ESI+) calcd for $\mathrm{C}_{25} \mathrm{H}_{25} \mathrm{~N}_{4} \mathrm{O}_{7}$ 493.1718; found $493.1703[\mathrm{M}+\mathrm{H}]$.

\section{Dimethyl}

6-amino-4-(3-chlorophenyl)-5-cyano-1-(2,4-dimethoxyphenyl)-1,4dihydropyridine-2,3-dicarboxylate (4ab)

Following the general procedure, compound $4 \mathbf{a b}$ was obtained after $72 \mathrm{~h}$ of reaction at $10{ }^{\circ} \mathrm{C}$ and was purified by column chromatography ( $n$-hexane:AcOEt 7:3 to $1: 1$ ), as a yellow solid in $82 \%$ yield $(39.7 \mathrm{mg})$. M.p. $102-106^{\circ} \mathrm{C}$. The ee of the product was determined to be $74 \%$ by HPLC using a Daicel Chiralpak IB column $\left(n\right.$-hexane $/ i$-PrOH $=70: 30$, flow rate $1 \mathrm{~mL} \mathrm{~min}{ }^{-1}$, $\lambda=238.0 \mathrm{~nm}): \tau_{\text {major }}=19.0 \mathrm{~min} ; \tau_{\text {minor }}=9.3 \mathrm{~min} .[\alpha]_{\mathrm{D}}^{22}=-4.7\left(c=0.21, \mathrm{CHCl}_{3}, 74 \%\right.$ ee $)$. 
${ }^{1} \mathrm{H}-\mathrm{NMR}\left(300 \mathrm{MHz}, \mathrm{DMSO}-d_{6}\right) \delta 3.32(\mathrm{~s}, 0.6 \mathrm{H}), 3.36(\mathrm{~s}, 2.4 \mathrm{H}), 3.50(\mathrm{~s}, 0.6 \mathrm{H}), 3.51(\mathrm{~s}, 2.4 \mathrm{H})$, $3.80(\mathrm{~s}, 0.6 \mathrm{H}), 3.81(\mathrm{~s}, 3 \mathrm{H}), 3.89(\mathrm{~s}, 2.4 \mathrm{H}), 4.46(\mathrm{~s}, 0.8 \mathrm{H}), 4.50(\mathrm{~s}, 0.2 \mathrm{H}), 5.62(\mathrm{br} \mathrm{s}, 2 \mathrm{H})$, 6.55-6.59 (m, 0.8H), 6.57-6.62 (m, 0.2H), $6.68(\mathrm{~d}, J=2.6 \mathrm{~Hz}, 0.2 \mathrm{H}), 6.71(\mathrm{~d}, J=2.6 \mathrm{~Hz}$, $0.8 \mathrm{H}), 7.11(\mathrm{~d}, J=8.6 \mathrm{~Hz}, 0.2 \mathrm{H}), 7.12(\mathrm{~d}, J=8.7 \mathrm{~Hz}, 0.8 \mathrm{H}), 7.23-7.47(\mathrm{~m}, 4 \mathrm{H}) .{ }^{13} \mathrm{C}-\mathrm{APT}-$ NMR (75 MHz, DMSO-d6) $\delta 38.8$ (1C), 51.7 (1C), 52.2 (1C), 55.5 (1C), 56.0 (1C), 58.7 (1C), 99.2 (1C), 103.0 (1C), 105.0 (1C), 115.7 (1C), 120.8 (1C), 126.0 (1C), 126.8 (1C), 127.0 (1C), 130.1 (1C), 132.2 (1C), 133.1 (1C), 142.9 (1C), 148.4 (1C), 151.0 (1C), 157.7 (1C), 161.7 (1C), 162.7 (1C), 164.8 (1C). IR (neat) $\left(\mathrm{cm}^{-1}\right) \vee 3465,3346,3221,3004,2950,2842$, $2180,1743,1705,1645,1609,1568,1508,1468,1432,1414,1355,1335,1310,1285,1260$, $1208,1162,1112,1078,1055,1026,972,934,829,806,777,767,730,709$. HRMS (ESI+) calcd for $\mathrm{C}_{24} \mathrm{H}_{21} \mathrm{ClN}_{3} \mathrm{O}_{6} 482.1113$; found 482.1100 [M-H].

\section{Diethyl}

6-amino-4-(3-chlorophenyl)-5-cyano-1-(2,4-dimethoxyphenyl)-1,4-

\section{dihydropyridine-2,3-dicarboxylate (4bb)}

Following the general procedure, compound $4 \mathbf{b b}$ was obtained after $72 \mathrm{~h}$ of reaction at $10{ }^{\circ} \mathrm{C}$ and was purified by column chromatography ( $n$-hexane:AcOEt 7:3 to 1:1), as a yellow solid in $98 \%$ yield $(50.2 \mathrm{mg})$. M.p. $75-78^{\circ} \mathrm{C}$. The ee of the product was determined to be $74 \%$ by HPLC using a Daicel Chiralpak IB column ( $n$-hexane $/ i$-PrOH $=80: 20$, flow rate $1 \mathrm{~mL} \mathrm{~min}{ }^{-1}$, $\lambda=254.0 \mathrm{~nm}): \tau_{\text {major }}=24.8 \mathrm{~min} ; \tau_{\text {minor }}=11.5 \mathrm{~min} .[\alpha]_{\mathrm{D}}{ }^{27}=-8.5\left(c=0.29, \mathrm{CHCl}_{3}, 74 \%\right.$ ee $)$. ${ }^{1} \mathrm{H}-\mathrm{NMR}\left(300 \mathrm{MHz}, \mathrm{DMSO}-d_{6}\right) \delta 0.88(\mathrm{t}, J=7.1 \mathrm{~Hz}, 0.6 \mathrm{H}), 0.89(\mathrm{t}, J=7.1 \mathrm{~Hz}, 2.4 \mathrm{H}), 1.03$ (t, $J=7.0 \mathrm{~Hz}, 0.6 \mathrm{H}), 1.05(\mathrm{t}, J=7.1 \mathrm{~Hz}, 2.4 \mathrm{H}), 3.81(\mathrm{~s}, 3.6 \mathrm{H}), 3.71-4.02(\mathrm{~m}, 4 \mathrm{H}), 3.91(\mathrm{~s}$, 2.4H), $4.47(\mathrm{~s}, 0.8 \mathrm{H}), 4.51(\mathrm{~s}, 0.2 \mathrm{H}), 5.60(\mathrm{br} \mathrm{s}, 2 \mathrm{H}), 6.55-6.59(\mathrm{~m}, 0.8 \mathrm{H}), 6.57-6.62(\mathrm{~m}$, $0.2 \mathrm{H}), 6.68(\mathrm{~d}, J=2.6 \mathrm{~Hz}, 0.2 \mathrm{H}), 6.72(\mathrm{~d}, J=2.6 \mathrm{~Hz}, 0.8 \mathrm{H}), 7.12(\mathrm{~d}, J=8.6 \mathrm{~Hz}, 0.2 \mathrm{H}), 7.13$ $(\mathrm{d}, J=8.7 \mathrm{~Hz}, 0.8 \mathrm{H}), 7.27-7.47(\mathrm{~m}, 4 \mathrm{H}) .{ }^{13} \mathrm{C}-\mathrm{APT}-\mathrm{NMR}\left(75 \mathrm{MHz}, \mathrm{DMSO}-d_{6}\right) \delta 13.2(1 \mathrm{C})$, 13.6 (1C), 38.9 (1C), 55.6 (1C), 56.0 (1C), 58.6 (1C), 60.3 (1C), 61.1 (1C), 99.2 (1C), 103.1 
(1C), 104.9 (1C), 115.7 (1C), 120.9 (1C), 126.2 (1C), 126.8 (1C), 127.2 (1C), 130.1 (1C), 132.5 (1C), 133.0 (1C), 142.7 (1C), 148.6 (1C), 151.1 (1C), 157.9 (1C), 161.8 (1C), 162.2 (1C), 164.3 (1C). IR (neat) $\left(\mathrm{cm}^{-1}\right)$ v 3461, 3345, 2983, 2937, 2181, 1738, 1699, 1647, 1609, $1567,1508,1470,1415,1391,1369,1344,1332,1309,1285,1260,1239,1205,1163,1106$, 1078, 1055, 1017, 937, 922, 888, 861, 828, 796, 777, 730, 716. HRMS (ESI+) calcd for $\mathrm{C}_{26} \mathrm{H}_{25} \mathrm{ClN}_{3} \mathrm{O}_{6} 510.1426$; found 510.1416 [M-H].

Dimethyl

6-amino-4-(4-chlorophenyl)-5-cyano-1-(2,4-dimethoxyphenyl)-1,4-

\section{dihydropyridine-2,3-dicarboxylate (4ac)}

Following the general procedure, compound 4ac was obtained after $72 \mathrm{~h}$ of reaction at $10{ }^{\circ} \mathrm{C}$ and was purified by column chromatography ( $n$-hexane:AcOEt 7:3 to $1: 1$ ), as a yellow solid in $36 \%$ yield (17.4 mg). M.p. $158-163{ }^{\circ} \mathrm{C}$. The ee of the product was determined to be $72 \%$ by HPLC using a Daicel Chiralpak IB column ( $n$-hexane $/ i-\mathrm{PrOH}=70: 30$, flow rate $1 \mathrm{~mL} \mathrm{~min}{ }^{-1}$, $\lambda=238.0 \mathrm{~nm}): \tau_{\text {major }}=17.6 \mathrm{~min} ; \tau_{\text {minor }}=9.1 \mathrm{~min} .[\alpha]_{\mathrm{D}}{ }^{25}=+10.1\left(c=0.20, \mathrm{CHCl}_{3}, 72 \%\right.$ ee $)$. ${ }^{1} \mathrm{H}-\mathrm{NMR}\left(400 \mathrm{MHz}, \mathrm{CDCl}_{3}\right) \delta 3.47(\mathrm{~s}, 0.75 \mathrm{H}), 3.50(\mathrm{~s}, 2.25 \mathrm{H}), 3.59$ (s, 0.75H), 3.59 (s, $2.25 \mathrm{H}), 3.85(\mathrm{~s}, 3 \mathrm{H}), 3.89(\mathrm{~s}, 0.75 \mathrm{H}), 3.90(\mathrm{~s}, 2.25 \mathrm{H}), 4.16($ br s, $1.5 \mathrm{H}), 4.19($ br s, $0.5 \mathrm{H}), 4.59$ $(\mathrm{s}, 0.75 \mathrm{H}), 4.66(\mathrm{~s}, 0.25 \mathrm{H}), 6.50(\mathrm{dd}, J=8.6 \mathrm{~Hz}, 2.6 \mathrm{~Hz}, 0.75 \mathrm{H}), 6.53-6.55(\mathrm{~m}, 1.25 \mathrm{H}), 7.16$ $(\mathrm{d}, J=8.0 \mathrm{~Hz}, 0.25 \mathrm{H}), 7.19(\mathrm{~d}, J=8.6 \mathrm{~Hz}, 0.75 \mathrm{H}), 7.29-7.36(\mathrm{~m}, 2.5 \mathrm{H}), 7.43-7.47(\mathrm{~m}, 1.5 \mathrm{H})$. ${ }^{13}$ C-APT-NMR (75 MHz, DMSO-d6) $\delta 38.4$ (1C), 51.7 (1C), 52.2 (1C), 55.5 (1C), 56.01 (1C), 58.9 (1C), 99.1 (1C), 103.3 (1C), 104.9 (1C), 115.8 (1C), 120.9 (1C), 128.3 (2C), 129.0 (2C), 131.4 (1C), 132.2 (1C), 142.8 (1C), 144.9 (1C), 151.0 (1C), 157.7 (1C), 161.7 (1C), $162.8(1 \mathrm{C}), 164.9$ (1C). IR (neat) $\left(\mathrm{cm}^{-1}\right) \vee 3313,3216,2947,2923,2852,2175,1752,1707$, $1649,1610,1568,1508,1492,1417,1357,1312,1285,1265,1243,1207,1160,1115,1089$, 1024, 1016, 978, 934, 918, 840, 822, 804, 777, 754, 730. HRMS (ESI+) calcd for $\mathrm{C}_{24} \mathrm{H}_{21} \mathrm{ClN}_{3} \mathrm{O}_{6} 482.1113$; found $482.1100[\mathrm{M}-\mathrm{H}]$. 


\section{dihydropyridine-2,3-dicarboxylate (4bd)}

Following the general procedure, compound $4 \mathbf{b d}$ was obtained after $72 \mathrm{~h}$ of reaction at $10{ }^{\circ} \mathrm{C}$ and was purified by column chromatography ( $n$-hexane:AcOEt 7:3 to 1:1), as a yellow solid in $96 \%$ yield (48.2 mg). M.p. $>110{ }^{\circ} \mathrm{C}$ decomp. The ee of the product was determined to be $74 \%$ by HPLC using a Daicel Chiralpak IB column $(n$-hexane $/ i-\mathrm{PrOH}=80: 20$, flow rate 1 $\left.\mathrm{mL} \min ^{-1}, \lambda=254.0 \mathrm{~nm}\right): \tau_{\text {major }}=31.3 \mathrm{~min} ; \tau_{\text {minor }}=17.3 \mathrm{~min} .[\alpha]_{\mathrm{D}}^{22}=+26.3\left(c=0.20, \mathrm{CHCl}_{3}\right.$, 74\% ee). ${ }^{1} \mathrm{H}-\mathrm{NMR}(300 \mathrm{MHz}, \mathrm{DMSO}-d 6) \delta 0.88(\mathrm{t}, J=7.1 \mathrm{~Hz}, 0.6 \mathrm{H}), 0.89(\mathrm{t}, J=7.1 \mathrm{~Hz}$, 2.4H), $1.00(\mathrm{t}, J=7.0 \mathrm{~Hz}, 0.6 \mathrm{H}), 1.02(\mathrm{t}, J=7.1 \mathrm{~Hz}, 2.4 \mathrm{H}), 3.81(\mathrm{~s}, 3.6 \mathrm{H}), 3.71-3.97(\mathrm{~m}, 4 \mathrm{H})$, $3.89(\mathrm{~s}, 2.4 \mathrm{H}), 4.54(\mathrm{~s}, 0.8 \mathrm{H}), 4.59(\mathrm{~s}, 0.2 \mathrm{H}), 5.64(\mathrm{br} \mathrm{s}, 2 \mathrm{H}), 6.57(\mathrm{dd}, J=8.7 \mathrm{~Hz}, J=2.7 \mathrm{~Hz}$ $0.8 \mathrm{H}), 6.59(\mathrm{dd}, J=8.7 \mathrm{~Hz}, J=2.7 \mathrm{~Hz}, 0.2 \mathrm{H}), 6.67(\mathrm{~d}, J=2.6 \mathrm{~Hz}, 0.2 \mathrm{H}), 6.72(\mathrm{~d}, J=2.6 \mathrm{~Hz}$, $0.8 \mathrm{H}), 7.13(\mathrm{~d}, J=8.7 \mathrm{~Hz}, 0.8 \mathrm{H}), 7.20(\mathrm{~d}, J=8.7 \mathrm{~Hz}, 0.2 \mathrm{H}), 7.48(\mathrm{~d}, J=8.3 \mathrm{~Hz}, 0.4 \mathrm{H}), 7.62$ $(\mathrm{d}, J=8.3 \mathrm{~Hz}, 1.6 \mathrm{H}), 7.87(\mathrm{~d}, J=8.3 \mathrm{~Hz}, 0.4 \mathrm{H}), 7.93(\mathrm{~d}, J=8.3 \mathrm{~Hz}, 1.6 \mathrm{H}) .{ }^{13} \mathrm{C}-\mathrm{APT}-\mathrm{NMR}$ (75 MHz, DMSO-d6) $\delta 13.2(1 \mathrm{C}), 13.6$ (1C), 39.2 (1C), 55.6 (1C), 56.0 (1C), 58.2 (1C), 60.3 (1C), 61.1 (1C), 99.1 (1C), 102.9 (1C), 104.9 (1C), 109.5 (1C), 115.7 (1C), 118.8 (1C), 120.7 (1C), 128.2 (2C), 132.4 (2C), 132.5 (1C), 143.1 (1C), 151.2 (1C), 151.3 (1C), 157.8 (1C), 161.9 (1C), 162.1 (1C), 164.1 (1C). IR (neat) $\left(\mathrm{cm}^{-1}\right) \vee 3458,3341,3219,2980,2936,2227$, $2181,1739,1704,1645,1606,1508,1463,1454,1440,1416,1392,1369,1327,1309,1286$, $1261,1207,1162,1105,1054,1025,936,922,900,856,828,803,786,770$. HRMS (ESI+) calcd for $\mathrm{C}_{27} \mathrm{H}_{25} \mathrm{~N}_{4} \mathrm{O}_{6} 501.1769$; found 501.1744 [M-H].

Dimethyl

6-amino-4-(4-bromophenyl)-5-cyano-1-(2,4-dimethoxyphenyl)-1,4dihydropyridine-2,3-dicarboxylate (4ae)

Following the general procedure, compound 4ae was obtained after $72 \mathrm{~h}$ of reaction at $10{ }^{\circ} \mathrm{C}$ and was purified by column chromatography ( $n$-hexane:AcOEt 7:3 to $1: 1$ ), as a yellow solid 
in $43 \%$ yield $(22.7 \mathrm{mg})$. M.p. $>95{ }^{\circ} \mathrm{C}$ decomp. The ee of the product was determined to be $76 \%$ by HPLC using a Daicel Chiralpak IB column $(n$-hexane $/ i-\mathrm{PrOH}=70: 30$, flow rate 1 $\left.\mathrm{mL} \min ^{-1}, \lambda=237.0 \mathrm{~nm}\right): \tau_{\text {major }}=18.6 \mathrm{~min} ; \tau_{\text {minor }}=9.4 \mathrm{~min} .[\alpha]_{\mathrm{D}}^{25}=+8.7\left(c=0.20, \mathrm{CHCl}_{3}\right.$, 76\% ee). ${ }^{1} \mathrm{H}-\mathrm{NMR}(300 \mathrm{MHz}, \mathrm{DMSO}-d 6) \delta 3.32(\mathrm{~s}, 0.75 \mathrm{H}), 3.36(\mathrm{~s}, 2.25 \mathrm{H}), 3.50(\mathrm{~s}, 3 \mathrm{H})$, $3.80(\mathrm{~s}, 0.75 \mathrm{H}), 3.81(\mathrm{~s}, 3 \mathrm{H}), 3.86(\mathrm{~s}, 2.25 \mathrm{H}), 4.42(\mathrm{~s}, 0.75 \mathrm{H}), 4.47(\mathrm{~s}, 0.25 \mathrm{H}), 5.57(\mathrm{br} \mathrm{s}, 2 \mathrm{H})$, $6.56(\mathrm{dd}, J=8.7 \mathrm{~Hz}, J=2.7 \mathrm{~Hz}, 0.75 \mathrm{H}), 6.60(\mathrm{dd}, J=8.6 \mathrm{~Hz}, J=2.6 \mathrm{~Hz}, 0.25 \mathrm{H}), 6.67(\mathrm{~d}, J=$ $2.6 \mathrm{~Hz}, 0.25 \mathrm{H}), 6.71(\mathrm{~d}, J=2.6 \mathrm{~Hz}, 0.75 \mathrm{H}), 7.12(\mathrm{~d}, J=8.7 \mathrm{~Hz}, 0.75 \mathrm{H}), 7.17(\mathrm{~d}, J=8.7 \mathrm{~Hz}$, 0.25H), 7.23-7.26 (m, 0.5H), 7.36-7.39 (m, 1.5H), 7.57-7.59 (m, 0.5H), 7.61-7.64 (m, 1.5H). ${ }^{13} \mathrm{C}-\mathrm{APT}-\mathrm{NMR}\left(75 \mathrm{MHz}, \mathrm{DMSO}-d_{6}\right) \delta 38.5$ (1C), 51.7 (1C), 52.2 (1C), 55.5 (1C), 56.1 (1C), 58.9 (1C), 99.2 (1C), 103.3 (1C), 104.9 (1C), 115.8 (1C), 119.9 (1C), 121.0 (1C), 129.4 (2C), 131.2 (2C), 132.2 (1C), 142.8 (1C), 145.3 (1C), 151.0 (1C), 157.7 (1C), 161.7 (1C), 162.8 (1C), 164.9 (1C). IR (neat) $\left(\mathrm{cm}^{-1}\right) \vee 3451,3353,3219,2950,2920,2849,2180,1744,1706$, $1646,1605,1568,1508,1455,1416,1344,1313,1285,1261,1208,1163,1108,1053,1026$, 972, 934, 875, 860, 821, 730, 711. HRMS (ESI+) calcd for $\mathrm{C}_{24} \mathrm{H}_{21} \mathrm{BrN}_{3} \mathrm{O}_{6}$ 526.0608; found $526.0596[\mathrm{M}-\mathrm{H}]$

\section{Dimethyl}

6-amino-5-cyano-1-(2,4-dimethoxyphenyl)-4-(3-nitrophenyl)-1,4-

\section{dihydropyridine-2,3-dicarboxylate (4af)}

Following the general procedure, compound 4af was obtained after $72 \mathrm{~h}$ of reaction at $10{ }^{\circ} \mathrm{C}$ and was purified by column chromatography ( $n$-hexane:AcOEt 7:3 to 1:1), as a yellow solid in $99 \%$ yield (48.9 mg). M.p. $>106{ }^{\circ} \mathrm{C}$ decomp. The ee of the product was determined to be $77 \%$ by HPLC using a Daicel Chiralpak IB column ( $n$-hexane $/ i-\mathrm{PrOH}=70: 30$, flow rate 1 $\left.\mathrm{mL} \min ^{-1}, \lambda=238.0 \mathrm{~nm}\right): \tau_{\text {major }}=23.9 \mathrm{~min} ; \tau_{\text {minor }}=12.4 \mathrm{~min} .[\alpha]_{\mathrm{D}}{ }^{24}=-3.0\left(c=0.21, \mathrm{CHCl}_{3}\right.$, 77\%). ${ }^{1} \mathrm{H}-\mathrm{NMR}\left(300 \mathrm{MHz}, \mathrm{DMSO}-d_{6}\right) \delta 3.33(\mathrm{~s}, 0.6 \mathrm{H}), 3.37$ (s, 2.4H), $3.50(\mathrm{~s}, 3 \mathrm{H}), 3.82$ (s, 3.6H), $3.85(\mathrm{~s}, 2.4 \mathrm{H}), 4.67(\mathrm{~s}, 0.8 \mathrm{H}), 4.68(\mathrm{~s}, 0.2 \mathrm{H}), 5.70($ br s, 2H), 6.54-6.59 (m, 0.8H), 
6.58-6.63 (m, 0.2H), $6.69(\mathrm{~d}, J=2.6 \mathrm{~Hz}, 0.2 \mathrm{H}), 6.72(\mathrm{~d}, J=2.6 \mathrm{~Hz}, 0.8 \mathrm{H}), 7.14(\mathrm{~d}, J=8.6$ $\mathrm{Hz}, 0.8 \mathrm{H}), 7.16(\mathrm{~d}, J=8.7 \mathrm{~Hz}, 0.2 \mathrm{H}), 7.75(\mathrm{t}, J=7.9 \mathrm{~Hz}, 1 \mathrm{H}), 7.75-7.80(\mathrm{~m}, 0.2 \mathrm{H}), 7.85-7.90$ (m, Hz, 0.8H), 8.10-8.23 (m, 2H). ${ }^{13} \mathrm{C}-\mathrm{APT}-\mathrm{NMR}\left(75 \mathrm{MHz}, \mathrm{DMSO}-d_{6}\right) \delta 38.8(1 \mathrm{C}), 51.8$ (1C), 52.3 (1C), 55.5 (1C), 56.1 (1C), 58.2 (1C), 99.2 (1C), 102.6 (1C), 105.0 (1C), 115.6 (1C), 120.7 (1C), 121.5 (1C), 121.9 (1C), 130.0 (1C), 132.2 (1C), 134.1 (1C), 143.3 (1C), 148.0 (1C), 148.1 (1C), 151.2 (1C), 157.7 (1C), 161.8 (1C), 162.6 (1C), 164.7 (1C). IR (neat) $\left(\mathrm{cm}^{-1}\right) \vee 3461,3361,3094,2947,2180,1744,1704,1649,1608,1567,1527,1506,1470$, $1415,1348,1314,1284,1262,1206,1164,1112,1057,1022,971,934,922,906,821,805$, 782, 766, 743, 730, 711. HRMS (ESI+) calcd for $\mathrm{C}_{24} \mathrm{H}_{21} \mathrm{~N}_{4} \mathrm{O}_{8} 493.1354$; found 493.1351 [M$\mathrm{H}]$.

\section{Dimethyl 6-amino-5-cyano-1-(2,4-dimethoxyphenyl)-4-p-tolyl-1,4-dihydropyridine-2,3- dicarboxylate (4ag)}

Following the general procedure, compound 4 ag was obtained after $72 \mathrm{~h}$ of reaction at $10^{\circ} \mathrm{C}$ and was purified by column chromatography ( $n$-hexane:AcOEt 7:3 to $1: 1$ ), as a yellow solid in $22 \%$ yield (10.2 mg). M.p. $123-126^{\circ} \mathrm{C}$. The ee of the product was determined to be $72 \%$ by HPLC using a Daicel Chiralpak IB column ( $n$-hexane $/ i-\mathrm{PrOH}=70: 30$, flow rate $1 \mathrm{~mL} \mathrm{~min}{ }^{-1}$, $\lambda=240.2 \mathrm{~nm}): \tau_{\text {major }}=15.1 \mathrm{~min} ; \tau_{\text {minor }}=8.5 \mathrm{~min} .[\alpha]_{\mathrm{D}}{ }^{26}=+7.6\left(c=0.20, \mathrm{CHCl}_{3}, 72 \%\right.$ ee $)$. ${ }^{1} \mathrm{H}-\mathrm{NMR}\left(300 \mathrm{MHz}, \mathrm{DMSO}-d_{6}\right) \delta 2.30$ (s, 3H), 3.32 (s, 0.9H), 3.36 (s, 2.1H), 3.50 (s, 3H), $3.82(\mathrm{~s}, 3.9 \mathrm{H}), 3.86(\mathrm{~s}, 2.1 \mathrm{H}), 4.38(\mathrm{~s}, 0.7 \mathrm{H}), 4.42(\mathrm{~s}, 0.3 \mathrm{H}), 5.38($ br s, 2H), 6.53-6.58 (m, $0.7 \mathrm{H}), 6.56-6.61(\mathrm{~m}, 0.3 \mathrm{H}), 6.68(\mathrm{~d}, J=2.5 \mathrm{~Hz}, 0.3 \mathrm{H}), 6.70(\mathrm{~d}, J=2.6 \mathrm{~Hz}, 0.7 \mathrm{H}), 7.12(\mathrm{~d}, J=$ $8.7 \mathrm{~Hz}, 1 \mathrm{H}), 7.19(\mathrm{~d}, J=8.2 \mathrm{~Hz}, 2.6 \mathrm{H}), 7.32(\mathrm{~d}, J=8.0 \mathrm{~Hz}, 1.4 \mathrm{H}) .{ }^{13} \mathrm{C}-\mathrm{APT}-\mathrm{NMR}(75 \mathrm{MHz}$, DMSO-d6) $\delta 20.4$ (1C), 38.4 (1C), 51.4 (1C), 52.0 (1C), 55.4 (1C), 55.8 (1C), 59.6 (1C), 99.1 (1C), 103.8 (1C), 104.9 (1C), 116.0 (1C), 120.9 (1C), 127.0 (2C), 128.7 (2C), 132.0 (1C), 135.6 (1C), 142.3 (1C), 142.8 (1C), 150.7 (1C), 157.7 (1C), 161.6 (1C), 162.8 (1C), 164.9 
(1C). IR (neat) $\left(\mathrm{cm}^{-1}\right) \vee 3445,3343,2947,2840,2180,1743,1705,1646,1609,1569,1508$, $1459,1435,1415,1389,1353,1329,1314,1285,1261,1208,1163,1110,1054,1024,973$, 933, 864, 835, 804, 788, 772, 735. HRMS (ESI+) calcd for $\mathrm{C}_{25} \mathrm{H}_{24} \mathrm{~N}_{3} \mathrm{O}_{6} 462.1660$; found 462.1662 [M-H].

\section{Dimethyl 6-amino-5-cyano-1-(2,4-dimethoxyphenyl)-4-phenyl-1,4-dihydropyridine-2,3- dicarboxylate (4ah)}

Following the general procedure, compound 4ah was obtained after $72 \mathrm{~h}$ of reaction at $10{ }^{\circ} \mathrm{C}$ and was purified by column chromatography ( $n$-hexane:AcOEt 7:3 to 1:1), as a yellow solid in $21 \%$ yield $(9.4 \mathrm{mg})$. M.p. $90-94{ }^{\circ} \mathrm{C}$. The ee of the product was determined to be $80 \%$ by HPLC using a Daicel Chiralpak IB column $\left(n\right.$-hexane $/ i$-PrOH $=70: 30$, flow rate $1 \mathrm{~mL} \mathrm{~min}{ }^{-1}$, $\lambda=238.0 \mathrm{~nm}): \tau_{\text {major }}=17.7 \mathrm{~min} ; \tau_{\text {minor }}=9.4 \mathrm{~min} .[\alpha]_{\mathrm{D}^{2}}=-13.6\left(c=0.17, \mathrm{CHCl}_{3}, 80 \%\right.$ ee $)$. ${ }^{1} \mathrm{H}-\mathrm{NMR}\left(300 \mathrm{MHz}, \mathrm{DMSO}-d_{6}\right) \delta 3.32(\mathrm{~s}, 0.75 \mathrm{H}), 3.36(\mathrm{~s}, 2.25 \mathrm{H}), 3.50(\mathrm{~s}, 3 \mathrm{H}), 3.81(\mathrm{~s}$, $3.75 \mathrm{H}), 3.86(\mathrm{~s}, 2.25 \mathrm{H}), 4.41(\mathrm{~s}, 0.75 \mathrm{H}), 4.45(\mathrm{~s}, 0.25 \mathrm{H}), 5.50(\mathrm{br} \mathrm{s}, 2 \mathrm{H}), 6.53-6.59(\mathrm{~m}$, $0.75 \mathrm{H}), 6.55-6.60(\mathrm{~m}, 0.25 \mathrm{H}), 6.68(\mathrm{~d}, J=2.6 \mathrm{~Hz}, 0.25 \mathrm{H}), 6.70(\mathrm{~d}, J=2.6 \mathrm{~Hz}, 0.75 \mathrm{H}), 7.12$ $(\mathrm{d}, J=8.7 \mathrm{~Hz}, 0.75 \mathrm{H}), 7.14(\mathrm{~d}, J=8.7 \mathrm{~Hz}, 0.25 \mathrm{H}), 7.22-7.29(\mathrm{~m}, 1.25 \mathrm{H}), 7.36-7.45(\mathrm{~m}$, 3.75H). ${ }^{13} \mathrm{C}-\mathrm{APT}-\mathrm{NMR}\left(75 \mathrm{MHz}, \mathrm{DMSO}-d_{6}\right) \delta 38.9$ (1C), 51.7 (1C), 52.2 (1C), 55.5 (1C), 56.0 (1C), 59.4 (1C), 99.2 (1C), 103.7 (1C), 104.9 (1C), 116.0 (1C), 121.1 (1C), 126.7 (1C), 127.2 (2C), 128.3 (2C), 132.2 (1C), 142.7 (1C), 145.9 (1C), 150.9 (1C), 157.7 (1C), 161.7 (1C), 162.9 (1C), 165.0 (1C). IR (neat) $\left(\mathrm{cm}^{-1}\right)$ v 3300, 3203, 3005, 2952, 2919, 2849, 2181, $1739,1707,1642,1609,1508,1454,1435,1419,1360,1286,1208,1160,1115,1076,1058$, $1025,974,933,861,835,793,756,728,699$. HRMS (ESI+) calcd for $\mathrm{C}_{24} \mathrm{H}_{22} \mathrm{~N}_{3} \mathrm{O}_{6} 448.1503$; found $448.1513[\mathrm{M}-\mathrm{H}]$. 


\section{dihydropyridine-2,3-dicarboxylate (4ai)}

Following the general procedure, compound 4ai was obtained after $72 \mathrm{~h}$ of reaction at $10{ }^{\circ} \mathrm{C}$ and was purified by column chromatography ( $n$-hexane:AcOEt 7:3 to 1:1), as a yellow solid in $15 \%$ yield $(6.8 \mathrm{mg})$. M.p. $139-141{ }^{\circ} \mathrm{C}$. The ee of the product was determined to be $76 \%$ by HPLC using a Daicel Chiralpak IB column $\left(n\right.$-hexane $/ i$-PrOH $=70: 30$, flow rate $1 \mathrm{~mL} \mathrm{~min}{ }^{-1}$, $\lambda=242.6 \mathrm{~nm}): \tau_{\text {major }}=14.2 \mathrm{~min} ; \tau_{\text {minor }}=9.7 \mathrm{~min} .[\alpha]_{\mathrm{D}}^{26}=-3.5\left(c=0.17, \mathrm{CHCl}_{3}, 76 \%\right.$ ee $)$. ${ }^{1} \mathrm{H}-\mathrm{NMR}\left(300 \mathrm{MHz}, \mathrm{DMSO}-d_{6}\right) \delta 3.32$ (s, 1.2H), 3.36 (s, 1.8H), 3.59 (s, 1.8H), $3.60(\mathrm{~s}, 1.2 \mathrm{H})$, $3.76(\mathrm{~s}, 1.8 \mathrm{H}), 3.81(\mathrm{~s}, 4.2 \mathrm{H}), 4.73(\mathrm{~s}, 0.6 \mathrm{H}), 4.77(\mathrm{~s}, 0.4 \mathrm{H}), 5.55$ (br s, 1.2H), 5.58 (br s, $0.8 \mathrm{H}), 6.56(\mathrm{td}, J=8.6 \mathrm{~Hz}, J=2.6 \mathrm{~Hz}, 1 \mathrm{H}), 6.64-6.70(\mathrm{~m}, 1 \mathrm{H}), 6.92(\mathrm{~d}, J=2.9 \mathrm{~Hz}, 0.4 \mathrm{H})$, 6.97-7.04 (m, 2H), $7.11(\mathrm{~d}, J=8.7 \mathrm{~Hz}, 0.6 \mathrm{H}), 7.37-7.41(\mathrm{~m}, 1 \mathrm{H}) .{ }^{13} \mathrm{C}-\mathrm{APT}-\mathrm{NMR}(75 \mathrm{MHz}$, DMSO-d6) $\delta 33.8$ (1C), 51.5 (1C), 52.0 (1C), 55.4 (1C), 55.6 (1C), 58.9 (1C), 99.1 (1C), 103.6 (1C), 104.8 (1C), 115.9 (1C), 120.8 (1C), 123.1 (1C), 124.0 (1C), 126.6 (1C), 131.8 (1C), 142.0 (1C), 149.5 (1C), 151.2 (1C), 157.6 (1C), 161.6 (1C), 162.6 (1C), 164.7 (1C). IR (neat) $\left(\mathrm{cm}^{-1}\right) \vee 3433,3330,3000,2947,2842,2182,1745,1710,1647,1609,1574,1508$, $1462,1434,1413,1351,1332,1313,1284,1210,1163,1105,1054,1029,971,933,852,825$, 803, 791, 773, 752, 730, 704. HRMS (ESI+) calcd for $\mathrm{C}_{22} \mathrm{H}_{20} \mathrm{~N}_{3} \mathrm{O}_{6} \mathrm{~S}$ 454.1067; found 454.1075 [M-H].

\section{Dimethyl}

6-amino-5-cyano-1-(2,4-dimethoxyphenyl)-4-(pyridin-3-yl)-1,4-

\section{dihydropyridine-2,3-dicarboxylate (4aj)}

Following the general procedure, compound 4aj was obtained after $72 \mathrm{~h}$ of reaction at $10{ }^{\circ} \mathrm{C}$ and was purified by column chromatography ( $n$-hexane:AcOEt 7:3 to 1:1), as a yellow solid in $79 \%$ yield (35.6 mg). M.p. $>185^{\circ} \mathrm{C}$ decomp. The ee of the product was determined to be $82 \%$ by HPLC using a Daicel Chiralpak IB column ( $n$-hexane $/ i-\mathrm{PrOH}=70: 30$, flow rate 1 
$\left.\mathrm{mL} \min ^{-1}, \lambda=238.7 \mathrm{~nm}\right): \tau_{\text {major }}=28.1 \mathrm{~min} ; \tau_{\text {minor }}=20.6 \mathrm{~min} .[\alpha]_{\mathrm{D}}{ }^{22}=-6.9\left(c=0.22, \mathrm{CHCl}_{3}\right.$, 82\% ee). ${ }^{1} \mathrm{H}-\mathrm{NMR}\left(300 \mathrm{MHz}, \mathrm{DMSO}-d_{6}\right) \delta 3.32$ (s, 0.6H), 3.36 (s, 2.4H), 3.49 (s, 0.6H), 3.51 (s, 2.4H), $3.81(\mathrm{~s}, 3.6 \mathrm{H}), 3.87(\mathrm{~s}, 2.4 \mathrm{H}), 4.47(\mathrm{~s}, 0.8 \mathrm{H}), 4.53(\mathrm{~s}, 0.2 \mathrm{H}), 5.67$ (br s, 2H), 6.53$6.59(\mathrm{~m}, 0.8 \mathrm{H}), 6.55-6.60(\mathrm{~m}, 0.2 \mathrm{H}), 6.67(\mathrm{~d}, J=2.6 \mathrm{~Hz}, 0.2 \mathrm{H}), 6.71(\mathrm{~d}, J=2.6 \mathrm{~Hz}, 0.8 \mathrm{H})$, $7.14(\mathrm{~d}, J=8.7 \mathrm{~Hz}, 0.8 \mathrm{H}), 7.20(\mathrm{~d}, J=8.7 \mathrm{~Hz}, 0.2 \mathrm{H}), 7.41-7.46(\mathrm{~m}, 0.2 \mathrm{H}), 7.45-7.50(\mathrm{~m}$, $0.8 \mathrm{H}), 7.61(\mathrm{dt}, J=8.0 \mathrm{~Hz}, J=2.0 \mathrm{~Hz}, 0.2 \mathrm{H}), 7.82(\mathrm{dt}, J=8.0 \mathrm{~Hz}, J=2.0 \mathrm{~Hz}, 0.8 \mathrm{H}), 8.48$ $(\mathrm{dd}, J=4.8 \mathrm{~Hz}, J=1.6 \mathrm{~Hz}, 1 \mathrm{H}), 8.48-8.50(\mathrm{~m}, 0.2 \mathrm{H}), 8.62-8.65(\mathrm{~m}, 0.8 \mathrm{H}) .{ }^{13} \mathrm{C}-\mathrm{APT}-\mathrm{NMR}$ (75 MHz, DMSO-d6) $\delta 36.7$ (1C), 51.8 (1C), 52.3 (1C), 55.6 (1C), 56.0 (1C), 58.4 (1C), 99.2 (1C), 103.1 (1C), 105.0 (1C), 115.8 (1C), 120.9 (1C), 123.8 (1C), 132.3 (1C), 134.7 (1C), 141.1 (1C), 143.1 (1C), 148.0 (1C), 148.6 (1C), 151.3 (1C), 157.6 (1C), 161.8 (1C), 162.8 (1C), 164.8, (1C). IR (neat) $\left(\mathrm{cm}^{-1}\right) \vee 3454,3346,3095,2947,2838,2178,1743,1698,1652$, $1611,1568,1529,1507,1478,1469,1452,1423,1353,1335,1314,1289,1261,1244,1219$, 1208, 1163, 1108, 1052, 1041, 1026, 971, 921, 862, 844, 826, 824, 802, 795, 781, 728, 714. HRMS (ESI+) calcd for $\mathrm{C}_{23} \mathrm{H}_{23} \mathrm{~N}_{4} \mathrm{O}_{6} 451.1612$; found 451.1625 [M+H].

\section{Author Information}

\section{Corresponding Authors}

*E-mail: mmaamarq@unizar.es and raquelph@unizar.es

\section{Notes}

The authors declare no competing financial interest.

Acknowledgment: Authors thank the Ministerio de Economía y Competitividad (MINECO/FEDER CTQ2016-75816-C2-1-P), the Spanish High Research Council (CSIC) (PIE-201580I010) and Gobierno de Aragón-Fondo Social Europeo (E77 and E104) for financial support of their research. 
Supporting Information Available: ${ }^{1} \mathrm{H}$ and ${ }^{13} \mathrm{C}$-APT NMR spectra of all new products. HPLC chromatograms for products $\mathbf{4}$ are also provided. This material is available free of charge via the Internet at http://pubs.acs.org.

\section{References}

1 Herrera, R. P. Top. Curr. Chem. 2016, 374, 29.

2 a) Reddy, G. M.; Shiradkar, M.; Chakravarthy, A. K. Curr. Org. Chem. 2007, 11, 847-852;

b) Edraki, N.; Mehdipour, A. R.; Khoshneviszadeh, M.; Miri, R. Drug Discov. Today 2009, 14, 1058-1066; c) Ioan, P.; Carosati, E.; Micucci, M.; Cruciani, G.; Broccatelli, F.; Zhorov, B. S.; Chiarini, A.; Budriesi, R. Curr. Med. Chem. 2011, 18, 4901-4922; d) Carosati, E.; Ioan, P.; Micucci, M.; Broccatelli, F.; Cruciani, G.; Zhorov, B. S.; Chiarini, A.; Budriesi, R. Curr. Med. Chem. 2012, 19, 4306-4323.

3 a) Goldmann, S.; Stoltefuss, J. Angew. Chem. Int. Ed. Engl. 1991, 30, 1559-1578; b) Shan, R.; Velazquez, C.; Knaus, E. E. J. Med. Chem. 2004, 47, 254-261; c) Carroll, W. A.; Altenbach, R. J.; Bai, H.; Brioni, J. D.; Brune, M. E.; Buckner, S. A.; Cassidy, C.; Chen, Y.; Coghlan, M. J.; Daza, A. V.; Drizin, I.; Fey, T. A.; Fitzgerald, M.; Gopalakrishnan, M.; Gregg, R. J.; Henry, R. F.; Holladay, M. W.; King, L. L.; Kort, M. E.; Kym, P. R.; Milicic, I.; Tang, R.; Turner, S. C.; Whiteaker, K. L.; Yi, L.; Zhang, H.; Sullivan, J. P. J. Med. Chem. 2004, 47, 3163-3179; d) Vo, D.; Matowe, W. C.; Ramesh, M.; Iqbal, N.; Wolowyk, M. W.; Howlett, S. E.; Knaus, E. E. J. Med. Chem. 1995, 38, 2851-2859; e) Sakai, T.; Teramura, T.; Okamiya, H.; Inagaki, O. Cardiovasc. Drug Rev. 1997, 15, 273-290.

4 Hantzsch, A. Ber. Dtsch. Chem. Ges. 1881, 14, 1637-1638.

5 a) Dondoni, A.; Massi, A. Acc. Chem. Res. 2006, 39, 451-463; b) Dondoni, A.; Massi, A.; Aldhoun, M. J. Org. Chem. 2007, 72, 7677-7687; c) Ducatti, D. R. B.; Massi, A.; Noseda, M. D.; Duarte, M. E. R.; Dondoni, A. Org. Biomol. Chem. 2009, 7, 1980-1986; d) Fustero, S.; 
Catalán, S.; Sánchez-Roselló, M.; Simón-Fuentes, A.; del Pozo, C. Org. Lett. 2010, 12, 34843487; e) Bonne, D.; Coquerel, Y.; Constantieux, T.; Rodriguez, J. Tetrahedron: Asymmetry 2010, 21, 1085-1109; f) Kwon, K.; Shin, J. A.; Lee, H.-Y. Tetrahedron 2011, 67, $10222-$ 10228; g) Bull, J. A.; Mousseau, J. J.; Pelletier, G.; Charette, A. B. Chem. Rev. 2012, 112, $2642-2713$

6 a) Wan, J.-P.; Liu, Y. $R S C A d v$. 2012, 2, 9763-9777; b) Pham, H. T.; Chataigner, I.; Renaud, J.-L. Curr. Org. Chem. 2012, 16, 1754-1775.

7 For some examples, see for instance: a) Dollé, F.; Hinnen, F.; Valette, H.; Fuseau, C.; Duval, R.; Péglion, J.-L.; Crouzel, C. Bioorg. Med. Chem. 1997, 5, 749-764; b) Zhang, B.-1.; He, W.; Shi, X.; Huan, M.-1.; Huang, Q.-j.; Zhou, S.-y. Bioorg. Med. Chem. Lett. 2010, 20, 805-808; c) Gotrane, D. M.; Deshmukh, R. D.; Ranade, P. V.; Sonawane, S. P.; Bhawal, B. M.; Gharpure, M. M.; Gurjar, M. K. Org. Process. Res. Dev. 2010, 14, 640-643.

8 a) Jian J.; Yu, J.; Sun, X.-X.; Rao, Q.-Q.; Gong, L.-Z. Angew. Chem. Int. Ed. 2008, 47, 2458-2462; b) Franke, P. T.; Johansen, R. L.; Bertelsen, S.; Jørgensen, K. A. Chem. Asian J. 2008, 3, 216-224; c) Yoshida, K.; Inokuma, T.; Takasu, K.; Takemoto, Y. Synlett 2010, $1865-$ 1869; d) Yoshida, K.; Inokuma, T.; Takasu, K.; Takemoto, Y. Molecules 2010, 15, 83058326; e) Noole, A.; Borissova, M.; Lopp, M.; Kanger, T. J. Org. Chem. 2011, 76, 1538-1545; f) Auria-Luna, F.; Marqués-López, E.; Mohammadi, S.; Heiran, R.; Herrera, R. P. Molecules 2015, 20, 15807-15826; g) Quinonero, O.; Jean, M.; Vanthuyne, N.; Roussel, C.; Bonne, D.; Constantieux, T.; Bressy, C.; Bugaut, X.; Rodriguez, J. Angew. Chem. Int. Ed. 2016, 55, 1401-1405.

9 a) Berkessel, A.; Gröger, H. Asymmetric Organocatalysis, Wiley-VCH Verlag GmbH: Weinheim, 2005; b) Enantioselective Organocatalysis, Dalko, P. I. (Ed.). Wiley-VCH Verlag GmbH: Weinheim, 2007; c) Comprehensive Enantioselective Organocatalysis, Dalko, P. I. (Ed.). Wiley-VCH Verlag GmbH: Weinheim, 2013. 
10 For racemic versions, see: a) Sun, J.; Xia, E.-Y.; Wu, Q.; Yan, C.-G. Org. Lett. 2010, 12, 3678-3681; b) Sun, J.; Sun, Y.; Xia, E.-Y.; Yan, C.-G. ACS Comb. Sci. 2011, 13, 436-441; c) Kiruthika, S. E.; Perumal, P. T. RSC Adv. 2014, 4, 3758-3767.

11 Efimova, S. V.; Korzhenevskii, A. B.; Koifman, O. I. Russian J. Gen. Chem. 2008, 78, $1614-1621$.

12 Choudhary, G.; Peddinti, R. K. Green Chem. 2011, 13, 3290-3299.

13 Postole, G.; Chowdhury, B.; Karmakar, B.; Pinki, K.; Banerji, J.; Auroux, A. J. Catal. 2010, 269, 110-121.

14 Hosseini-Sarvari, M.; Sharghi, H.; Etemad, S. Chin. J. Chem. 2007, 25, 1563-1567.

15 Kharas, G. B.; Hanawa, E.; Lachenberg, J.; Brozek, B.; Miramon, P.; Mojica, A. C.; Colbert, A. C.; Crowell, B. T.; Madison, A.; Martinez, A. P. J. Macromol. Sci. A 2008, 45, $420-424$

16 Yamashita, K.; Tanaka, T.; Hayashi, M. Tetrahedron 2005, 61, 7981-7985.

17 Ying, A.-G.; Wang, L.-M.; Wang, L.-L.; Chen, X.-Z.; Ye, W.-D. J. Chem. Res. 2010, 34, $30-33$ 